\title{
Revisiting the SUSY $\mu$ problem and its solutions in the LHC era
}

\author{
Kyu Jung Bae, ${ }^{1, *}$ Howard Baer, ${ }^{2, \dagger}$ Vernon Barger, ${ }^{3, \star}$ and Dibyashree Sengupta ${ }^{2, \S}$ \\ ${ }^{1}$ Center for Theoretical Physics of the Universe, Institute for Basic Science (IBS), Daejeon 34126, Korea \\ ${ }^{2}$ Homer L. Dodge Department of Physics and Astronomy, University of Oklahoma, \\ Norman, Oklahoma 73019, USA \\ ${ }^{3}$ Department of Physics, University of Wisconsin, Madison, Wisconsin 53706, USA
}

(Received 5 March 2019; published 21 June 2019)

\begin{abstract}
The supersymmetry (SUSY)-preserving $\mu$ parameter in SUSY theories is naively expected to be of order the Planck scale while phenomenology requires it to be of order the weak scale. This is the famous SUSY $\mu$ problem. Its solution involves two steps: first forbid $\mu$, perhaps via some symmetry, and then regenerate it of order the scale of soft SUSY-breaking terms. However, present LHC limits suggest the soft breaking scale $m_{\text {soft }}$ lies in the multi-TeV regime while naturalness requires $\mu \sim m_{W, Z, h} \sim 100 \mathrm{GeV}$ so that a little hierarchy (LH) appears with $\mu \ll m_{\text {soft }}$. We review 20 previously devised solutions to the SUSY $\mu$ problem and reevaluate them in light of whether they are apt to support the LH. We organize the 20 solutions as follows: (1) solutions from supergravity/superstring constructions, (2) extended minimal supersymmetric Standard Model solutions, (3) solutions from an extra local $U(1)^{\prime}$ and (4) solutions involving Peccei-Quinn symmetry and axions. Early solutions invoked a global Peccei-Quinn symmetry to forbid the $\mu$ term while relating the $\mu$ solution to solving the strong $C P$ problem via the axion. We discuss the gravity-safety issue pertaining to global symmetries and the movement instead toward local gauge symmetries or $R$ symmetries, either continuous or discrete. At present, discrete $R$ symmetries of order $M\left(\mathbb{Z}_{M}^{R}\right)$ which emerge as remnants of the Lorentz symmetry of compact dimensions seem favored. Even so, a wide variety of regenerative mechanisms are possible, some of which relate to other issues such as the strong $C P$ problem or the generation of neutrino masses. We also discuss the issue of the experimental verification or falsifiability of various solutions to the $\mu$ problem. Almost all solutions seem able to accommodate the LH.
\end{abstract}

DOI: $10.1103 /$ PhysRevD.99.115027

\section{INTRODUCTION: REFORMULATING THE $\mu$ PROBLEM FOR THE LHC ERA}

Supersymmetry (SUSY) provides a solution to the big hierarchy problem-why does the Higgs mass not blow up to the GUT/Planck scale?-via a neat cancellation of quadratic divergences which is required by extending the Poincaré group of spacetime symmetries to its maximal structure $[1,2]$. SUSY is also supported indirectly via the confrontation of data with virtual effects in that 1) the measured gauge couplings unify under minimal supersymmetric Standard Model (MSSM) renormalization group evolution [3], 2) the measured value of $m_{t}$ falls in the range required for a radiatively driven breakdown of electroweak

\footnotetext{
*kyujungbae@ibs.re.kr

tbaer@nhn.ou.edu

tbarger@pheno.wisc.edu

${ }^{\S}$ Dibyashree.Sengupta-1@ou.edu
}

Published by the American Physical Society under the terms of the Creative Commons Attribution 4.0 International license. Further distribution of this work must maintain attribution to the author(s) and the published article's title, journal citation, and DOI. Funded by SCOAP. symmetry [4], 3) the measured value of the Higgs boson mass falls squarely within the narrow allowed range required by the MSSM $[5,6]$ and 4) the measured values of $m_{W}$ and $m_{t}$ favor the MSSM with heavy superpartners [7]. In spite of these successes, so far no direct signal for SUSY has emerged at the LHC leading to the mass limits $m_{\tilde{g}} \gtrsim 2 \mathrm{TeV}$ and $m_{\tilde{t}_{1}} \gtrsim 1 \mathrm{TeV}$ while the rather large value of $m_{h} \simeq 125 \mathrm{GeV}$ also seemingly requires multi-TeV highly mixed top squarks [6]. The new LHC Higgs mass measurement and sparticle mass limits seem to have exacerbated the so-called little hierarchy problem (LHP) [8]: why does the Higgs mass not blow up to the soft SUSY-breaking scale $m_{\text {soft }} \gtrsim$ several TeV, or what stabilizes the apparent hierarchy $m_{h} \ll m_{\text {soft }}$ ? The LHP opens up the naturalness question: how can it be that the weak scale $m_{\text {weak }} \sim m_{W, Z, h} \sim 100 \mathrm{GeV}$ without unnatural fine-tunings of dimensionful terms in the MSSM Lagrangian?

The most direct link between the magnitude of the weak scale and the SUSY Lagrangian comes from minimization of the MSSM Higgs potential to determine the Higgs field vacuum expectation values (VEVs) [2]. A straightforward calculation [2] reveals that 


$$
\begin{aligned}
m_{Z}^{2} / 2 & =\frac{m_{H_{d}}^{2}+\Sigma_{d}^{d}-\left(m_{H_{u}}^{2}+\Sigma_{u}^{u}\right) \tan ^{2} \beta}{\tan ^{2} \beta-1}-\mu^{2} \\
& \simeq-m_{H_{u}}^{2}-\Sigma_{u}^{u}\left(\tilde{t}_{1,2}\right)-\mu^{2}
\end{aligned}
$$

where $\tan \beta \equiv v_{u} / v_{d}$ is the ratio of Higgs field VEVs, $\mu$ is the SUSY-conserving Higgs/Higgsino mass term and $m_{H_{u, d}}^{2}$ are soft SUSY-breaking up- and down-Higgs mass terms. The $\Sigma_{u}^{u}$ and $\Sigma_{d}^{d}$ terms contain a large assortment of loop corrections (see the Appendix of Ref. [9] for expressions) the largest of which are usually the $\Sigma_{u}^{u}\left(\tilde{t}_{1,2}\right)$ from the top-squark sector.

We can see immediately from the right-hand-side of Eq. (1) that if say one contribution is far larger than $m_{Z}^{2} / 2$, then another (unrelated) term will have to be fine-tuned to compensate so as to maintain $m_{Z}$ at its measured value. The electroweak (EW) fine-tuning measure $\Delta_{\mathrm{EW}}$ has been introduced $[9,10]$,

$$
\left.\Delta_{\mathrm{EW}} \equiv \max \mid \text { largest term on rhs of Eq.(1)|/( } m_{Z}^{2} / 2\right)
$$

to quantify the weak-scale fine-tuning required to maintain $m_{Z}$ at its measured value. While a low value of $\Delta_{\mathrm{EW}}$ seems to be a necessary condition for naturalness within the MSSM, the question is: is it also sufficient? It was argued in Refs. [11-14] that for correlated (i.e., interdependent) soft terms as should occur in any more fundamental theory such as supergravity (SUGRA) with a well-specified SUSYbreaking sector, or in string theory, other measures such as $\Delta_{\mathrm{HS}} \simeq \delta m_{h}^{2} / m_{h}^{2}$ and $\Delta_{\mathrm{BG}} \equiv \max _{i}\left|\frac{\partial \log m_{Z}^{2}}{\partial \log p_{i}}\right|$ (where the $p_{i}$ are fundamental model parameters) collapse to $\Delta_{\mathrm{EW}}$ so that $\Delta_{\mathrm{EW}}$ is sufficient as both an infrared and ultraviolet (UV) fine-tuning measure. In contrast, theories with multiple independent soft parameters may be susceptible to further fine-tunings which would otherwise cancel in a more fundamental theory. It should be recalled that in the multi-soft-parameter effective theories such as constrained minimal supersymmetric standard model/minimal supergravity Model, non-universal higgs model (with 2 extra parameters) etc., the various soft parameters are introduced to parametrize one's ignorance of the SUSY-breaking sector such that some choice of soft parameters will reflect the true choice in nature. However, in no sense are the multi-soft-parameter theories expected to be fundamental. Thus, in this paper we will adopt $\Delta_{\mathrm{EW}}$ as a measure of naturalness in fundamental theories with the MSSM as the weak scale effective theory. In Ref. [15], it was shown that the fine-tuning already turns on for values of $\Delta_{\mathrm{EW}} \sim 20-30$. We will adopt a value of $\Delta_{\mathrm{EW}}<30$ as a conservative choice for natural models of SUSY.

For a natural theory-where $m_{W, Z, h} \sim 100 \mathrm{GeV}$ because the rhs contributions to Eq. (1) are comparable to or less than the measured value of $m_{Z}^{2} / 2$-then evidently

(i) $m_{H_{u}}^{2}$ (weak) $\sim-(100-300)^{2} \mathrm{GeV}^{2}$

(ii) $|\mu| \sim 100-300 \mathrm{GeV}[16,17]$, and

(iii) the largest of the radiative corrections [usually $\left.\Sigma_{u}^{u}\left(\tilde{t}_{1,2}\right)\right]$ are not too large.
The first of these conditions pertains to the soft SUSYbreaking sector. It can be achieved for multi-TeV values of high-scale soft terms (as required by LHC limits) by radiatively driving $m_{H_{u}}^{2}$ from large, seemingly unnatural high scale values to a natural value at the weak scale. Thus, a high scale value of $m_{H_{u}}^{2}\left(\Lambda=m_{\mathrm{GUT}}\right)$ must be selected such that electroweak symmetry is barely broken. While this may seem to be a tuning in itself, such a selection seems to automatically emerge from SUSY within the string-landscape picture $[18,19]$. In this scenario, there is a statistical attraction towards large soft terms which must be balanced by the anthropic requirement that EW symmetry be properly broken and with a weak-scale magnitude not too far from its measured value[20]. The balance between these two tendencies pulls $m_{H_{u}}^{2}\left(m_{\mathrm{GUT}}\right)$ to such large values that EW symmetry is barely broken.

The third of the above conditions-that $\Sigma_{u}^{u}\left(\tilde{t}_{1,2}\right) \sim$ $100-300 \mathrm{GeV}$ - is achieved for third-generation squark soft terms in the several-TeV range along with a large trilinear soft term $A_{t}$ (as is expected in gravity-mediation models). These same conditions which reduce the $\Sigma_{u}^{u}\left(\tilde{t}_{1,2}\right)$ values also increase the Higgs mass to its measured value $m_{h} \sim 125 \mathrm{GeV}[9,10]$.

The second condition - that the superpotential $\mu$ parameter is of order the weak scale-brings up the famous SUSY $\mu$ problem [21]: since $W_{\text {MSSM }} \ni \mu H_{u} H_{d}$ is SUSY preserving, naively one expects the dimensionful parameter $\mu$ to be of order $m_{P} \simeq 2.4 \times 10^{18} \mathrm{GeV}$ while phenomenology requires $\mu \sim m_{\text {weak }}$. In this paper, we focus attention on the SUSY $\mu$ problem as occurs in gravity mediation. The SUSY $\mu$ problem in gauge-mediated supersymmetry breaking (GMSB) was summarized in Ref. [22]. In GMSB, since the trilinear soft terms are expected to be tiny, sparticle masses must become huge with highly unnatural contributions to the weak scale in order to accommodate a light Higgs boson with $m_{h} \simeq 125 \mathrm{GeV}[23,24] .{ }^{1}$

There are two parts to solving the SUSY $\mu$ problem:

(i) First, one must forbid the appearance of $\mu$, usually via some symmetry such as Peccei-Quinn (PQ) symmetry or better a continuous or discrete gauge or $R$ symmetry, and then

(ii) regenerate $\mu$ at the much lower weak scale $|\mu| \sim$ 100-300 GeV (the lower the more natural) via some mechanism such as symmetry breaking.

Many solutions to the SUSY $\mu$ problem have been proposed, and indeed in Sec. II we will review 20 of these. In most of these solutions, the goal (for gravity mediation) was to regenerate $\mu \sim m_{3 / 2}$ where $m_{3 / 2}$ is the gravitino mass which arises from SUGRA breaking and which sets the mass scale for the soft SUSY-breaking terms [28]. When many of these $\mu$ solutions were proposed-well before the

\footnotetext{
${ }^{1}$ We also do not consider SUSY models with nonholonomic soft terms [25] or multiple $\mu$ terms; it is not clear whether such models have viable UV completions [26,27].
} 
LHC era-it was commonly accepted that $m_{3 / 2} \sim m_{\text {weak }}$ which would also solve the SUSY naturalness problem. However, in light of the above discussion, the SUSY $\mu$ problem needs a reformulation for the LHC era: any solution to the SUSY $\mu$ problem should first forbid the appearance of $\mu$, but then regenerate it at the weak scale, which is now hierarchically smaller than the soft breaking scale:

$$
\begin{aligned}
|\mu| & \sim m_{\text {weak }} \sim 100-300 \mathrm{GeV} \ll m_{\text {soft }} \\
& \sim \text { multi }-\mathrm{TeV} \lesssim m_{3 / 2} .
\end{aligned}
$$

Our goal in this paper is to review various proposed solutions to the SUSY $\mu$ problem and confront them with the little hierarchy as established by LHC data and as embodied by Eq. (3). While many solutions can be tuned to maintain the little hierarchy, others may offer compatibility with or even a mechanism to generate Eq. (3). Thus, present LHC data may be pointing to favored solutions to the SUSY $\mu$ problem which may be reflective of the way nature actually works.

With this end in mind, in Sec. II we will review a variety of mechanisms which have been offered as solutions to the SUSY $\mu$ problem. We organize the 20 solutions according to:

(1) solutions from supergravity/superstring constructions,

(2) extended MSSM solutions,

(3) solutions from an extra local $U(1)^{\prime}$ and

(4) solutions involving PQ symmetry and axions.

Many of these solutions tend to relate the $\mu$ parameter to the scale of soft SUSY breaking which would place the $\mu$ parameter well above the weak scale and thus require significant EW fine-tuning. One such example is the original Kim-Nilles (KN) [29] model (Sec. II D 1) which generates a $\mu$ parameter $\mu \sim v_{\mathrm{PQ}}^{2} / m_{P}$ and relates $v_{\mathrm{PQ}} \sim m_{\text {hidden }}$ (where $m_{\text {hidden }}$ is a mass scale associated with hidden-sector SUGRA breaking) and thus obtains $\mu \sim v_{\mathrm{PQ}}^{2} / m_{P} \sim m_{\text {hidden }}^{2} /$ $m_{P} \sim m_{3 / 2}$. However, the LHP can also be accommodated by allowing for $v_{\mathrm{PQ}} \ll m_{\text {hidden }}$ so that $\mu \ll m_{3 / 2}$. While $\mathrm{KN}$ allows this possibility to be implemented "by hand," the later Murayama-Suzuki-Yanagida (MSY) [30], Choi-Chun-Kim (CCK) [31] and Stephen P. Martin (SPM) [32] models (Sec. II D 7) implement radiative PQ breaking as a consequence of SUSY breaking with the result that $v_{\mathrm{PQ}} \ll m_{\text {hidden }}$ and hence $\mu \ll m_{\text {soft }}$ [33].

A prominent criticism of the $\mu$ solutions based on the existence of a global PQ or discrete symmetry is that such symmetries are incompatible with gravity at high scales [34-38], i.e., that including the presence of gravity could spoil any global or discrete symmetries which may be postulated. In Sec. II D 4, we discuss possible ways around the gravity spoliation of global or discrete symmetries. The Martin-Babu-Gogoladze-Wang (MBGW) model [39] (Sec. II D 9) adopts a gravity-safe PQ symmetry thanks to a more fundamental discrete gauge symmetry $\mathbb{Z}_{22}$ and also generates PQ breaking from SUSY breaking, albeit not radiatively.
An attractive alternative to the discrete or continuous gauge symmetry resides in the possibility of a discrete or continuous $R$ symmetry. Several discrete $R$ symmetries are possible which are anomaly free (up to a Green-Schwarz term), forbid the $\mu$ parameter and other dangerous proton decay operators, and are consistent with an underlying grand unification structure [40,41]. Such discrete $R$ symmetries are expected to arise from compactification of extra dimensions in string theory. The $\mathbb{Z}_{4}^{R}$ symmetry stands out as a particularly simple approach that also leads to exact $R$-parity conservation. To relate a gravity-safe PQ solution to the strong $C P$ problem with a solution to the $\mu$ problem, we examine two hybrid models based on $\mathbb{Z}_{24}^{R}$ (Sec. II E). In this case, the PQ symmetry arises as an accidental approximate global symmetry which emerges from the more fundamental discrete $R$ symmetry. Here, the PQ breaking is generated through a large negative soft term and not radiatively.

In Sec. III we discuss the issue of the experimental testability and distinguishability of various solutions to the $\mu$ problem. In Sec. IV, we present a convenient table (Table XIV) which summarizes our review. Then we draw some final conclusions. Some pedagogical reviews providing an in-depth overview of supersymmetric models of particle physics can be found in Ref. [2].

\section{A REVIEW OF SOME SOLUTIONS TO THE SUSY $\mu$ PROBLEM}

In this section, we review some solutions to the SUSY $\mu$ problem. In the solutions reviewed here, the $\mu$ term is typically generated by breaking the symmetry which originally prohibits the $\mu$ term at the tree level. Depending on the source of such symmetry breaking, we categorize the solutions according to 1 . those from supergravity/superstring models, 2. those from (visible-sector) extensions of the MSSM, 3. those including an extra local $U(1)^{\prime}$ and 4. those which include also a solution to the strong $C P$ problem with Peccei-Quinn symmetry breaking.

\section{A. Solutions in supergravity/string constructions}

\section{Giudice-Masiero (GM)}

In supergravity models the Kähler function $G=K+$ $\log |W|^{2}$ is written in terms of the real Kähler potential $K$ and the holomorphic superpotential $W$. If we posit some symmetry (PQ or $R$ symmetry was suggested in Ref. [42]) to forbid the usual MSSM $\mu$ term, then one may regenerate it via the Higgs fields coupling to hidden-sector fields $h_{m}$ via nonrenormalizable terms in $K$ [42]:

$$
K \ni H_{u}^{\dagger} H_{u}+H_{d}^{\dagger} H_{d}+\left(\frac{\lambda_{\mu}}{m_{P}} H_{u} H_{d} h^{\dagger}+\text { H.c. }\right) .
$$

If we arrange for SUSY breaking in the hidden sector, then the auxiliary component of $h$ develops a $\operatorname{VEV}\left\langle F_{h}\right\rangle \sim m_{\text {hidden }}^{2}$ 
so that the gravitino gets a mass $m_{3 / 2} \sim m_{\text {hidden }}^{2} / m_{P}$. A $\mu$ term is generated of order

$$
\mu_{\mathrm{eff}}=\lambda_{\mu} \frac{\left\langle F_{h}^{*}\right\rangle}{m_{P}} \sim \lambda_{\mu} m_{\mathrm{hidden}}^{2} / m_{P} \sim \lambda_{\mu} m_{3 / 2} \sim m_{\mathrm{soft}} .
$$

Thus, in the GM case, the $\mu$ parameter arises which is typically of order the soft breaking scale unless the coupling $\lambda_{\mu}$ is suppressed at the $\sim 0.01-0.1$ level. $^{2}$

\section{Casas-Muñoz (CM)}

Casas and Muñoz [44] proposed a string-theory-inspired solution to the SUSY $\mu$ problem. In string theory, dimensionful couplings such as $\mu$ are already forbidden by the scale invariance of the theory so no new symmetries are needed to forbid it. They began with a superpotential of the form

$$
W=W_{0}+\lambda_{\mu} W_{0} H_{u} H_{d} / m_{P}^{2}
$$

where $W_{0}$ is the usual superpotential of the MSSM (but without the $\mu$ term) along with the hidden-sector component which is responsible for SUSY breaking: $W_{0}=$ $W_{0}^{\text {vis }}\left(z_{i}\right)+W_{0}^{\text {hid }}\left(h_{m}\right)$ where the $z_{i}$ comprise visible-sector fields while the $h_{m}$ denote hidden-sector fields. While the scale-variant $\mu$ term is forbidden in $W_{0}^{\text {vis }}$, the nonrenormalizable contribution in Eq. (6) is certainly allowed and, absent any symmetries which could forbid it, probably mandatory. Under, for instance, $F$-term SUSY breaking in the hidden sector, $W_{0}^{\text {hid }}$ gains a VEV $\left\langle W_{0}^{\text {hid }}\right\rangle \sim m_{\text {hidden }}^{2} m_{P}$ [as is easy to see in the simplest Polonyi model for SUSY breaking with $W_{\text {Polonyi }}=m_{\text {hidden }}^{2}\left(h+\beta m_{P}\right)$ where $\beta$ is a dimensionless constant]. Under these conditions, a $\mu$ term develops with

$$
\mu_{\text {eff }} \sim \lambda_{\mu} m_{\text {hidden }}^{2} / m_{P} \sim \lambda_{\mu} m_{3 / 2} \sim m_{\text {soft }} .
$$

Reference [44] went on to show that the CM solution can easily emerge in models of SUSY breaking due to hiddensector gaugino condensation at some intermediate mass scale $\Lambda_{h}$ (where then we would associate $m_{\text {hidden }}^{2} \simeq \Lambda_{h}^{3} / m_{P}$ ).

A benefit of the CM solution is that it should be consistent with any stringy UV completion [45] as it avoids the presence of some global (PQ) symmetry. A possible drawback to $\mathrm{CM}$ is that the $\mu$ term is naturally expected to be of order $m_{\text {soft }}$ instead of $m_{\text {weak }}$ unless $\lambda_{\mu}$ is suppressed (as in GM). One way to falsify the CM solution would be to discover a Dine-Fischler-Srednicki-Zhitnitsky (DFSZ)-like axion with consistent mass and coupling values. Such a discovery would exclude the second term in Eq. (6) since it would violate the PQ symmetry.

\footnotetext{
${ }^{2}$ Some recent work on gravity-mediated SUSY breaking and the GM solution to the $\mu$ problem is included in Ref. [43].
}

\section{3. $\mu$ and a big hierarchy from approximate $R$ symmetry}

In string theory models, approximate $R$ symmetries are expected to develop from the overall Lorentz symmetry of the ten-dimensional spacetime when compactified to four dimensions. Under a continuous $U(1)_{R}$ symmetry, the superspace coordinates transform nontrivially and hence so do the bosonic and fermionic components of superfields. Thus, these symmetries can be linked to the overall Lorentz symmetry where also bosons and fermions transform differently.

Under exact $R$ symmetry and supersymmetry, the superpotential $\mu$ term is forbidden since the gauge-invariant bilinear term of the Higgs pair $H_{u} H_{d}$ carries zero $R$ charge while the superpotential must have $R_{W}=+2$. However, $H_{u} H_{d}$ may couple to various other superfields $\phi_{i}$ which carry nontrivial $R$ charges so that

$$
W \ni P_{\mu}\left(\phi_{i}\right) H_{u} H_{d}
$$

where $P_{\mu}\left(\phi_{i}\right)$ is a sum over monomials in the fields $\phi_{i}^{n}$. Unbroken $R$ symmetry requires a vanishing $\left\langle P_{\mu}\left(\phi_{i}\right)\right\rangle$ but if the $R$ symmetry is approximate then nonvanishing $P_{\mu}\left(\phi_{i}\right)$ contributions will develop at higher orders in powers of the field VEVs $\left\langle\left(\phi_{i} / m_{P}\right)\right\rangle \lesssim 1$. Thus, a mild hierarchy in the field VEVs $\left\langle\phi_{i} / m_{P}\right\rangle \lesssim 1$, when raised to higher powers $\left\langle\left(\phi_{i} / m_{P}\right)^{n_{i}}\right\rangle \ll 1$, can generate a much larger hierarchy of scales [46]. ${ }^{3}$ In this solution to the $\mu$ problem, which is essentially a UV completion of the CM solution, $\mu \sim$ $m_{3 / 2} \sim\langle W\rangle$ is expected to arise.

\section{Solution via the discrete $R$ symmetry $\mathbb{Z}_{4}^{R}$}

A particularly attractive way to solve the $\mu$ problem in some string constructions is via a discrete Abelian $R$ symmetry $\mathbb{Z}_{4}^{R}$ [48-50]. Such $R$ symmetries may arise as discrete remnants of the Lorentz symmetry of extra-dimensional $(d=10)$ models upon compactification to $d=4$. In Ref. [51], the $\mathbb{Z}_{4}^{R}$ symmetry was invoked to forbid the $\mu$ term as well as dimension-four baryon- and lepton-numberviolating operators while dangerous dimension-five operators leading to proton decay are highly suppressed [40,41]. The desirable Weinberg neutrino mass operator is allowed. The $\mathbb{Z}_{4}^{R}$ charges are assigned so that all anomalies cancel by including Green-Schwarz terms (and extra $R$-charged singlets for gravitational anomalies). The $R$-charge assignments for the discrete $R$ symmetry $\mathbb{Z}_{4}^{R}$ are shown in the second row of Table I.

The charge assignments are consistent with embedding the matter superfields into a single 16 of $S O(10)$ while the split Higgs multiplets would arise from Wilson-line breaking of gauge symmetry. The $\mathbb{Z}_{4}^{R}$ symmetry may be broken via nonperturbative effects such as gaugino condensation breaking of SUGRA in the hidden sector so that a gravitino

\footnotetext{
${ }^{3}$ See also Ref. [47].
} 
TABLE I. $\mathbb{Z}_{4}^{R}$ charge assignments for various superfields of the Lee-Raby-Ratz-Ross-Schieren-Schmidt-Hoberg-Vaudrevange model [40].

\begin{tabular}{lcccccccc}
\hline \hline Multiplet & $H_{u}$ & $H_{d}$ & $Q_{i}$ & $L_{i}$ & $U_{i}^{c}$ & $D_{i}^{c}$ & $E_{i}^{c}$ & $N_{i}^{c}$ \\
\hline $\mathbb{Z}_{4}^{R}$ charge & 0 & 0 & 1 & 1 & 1 & 1 & 1 & 1 \\
\hline \hline
\end{tabular}

mass $m_{3 / 2}$ is induced along with soft terms $m_{\text {soft }} \sim m_{3 / 2}$. A $\mu$ term may arise via GM (Sec. II A 1) and/or CM (Sec. II A 2) so that $\mu \sim\langle W\rangle / m_{P}^{2} \sim m_{3 / 2} \sim m_{\text {soft. }}$. Although the discrete $\mathbb{Z}_{4}^{R} R$ symmetry is broken, the discrete matter $/ R$ parity remains unbroken so that the lightest supersymmetric particle (LSP) remains absolutely stable. This sort of solution to the $\mu$ problem is expected to be common in heterotic string models compactified on an orbifold [41]. Other possibilities for $\mathbb{Z}_{N}^{R}$ with $N>4$ also occur [41] and in fact any $N$ value is possible under anomaly cancellations provided one includes additional exotic matter into the visible sector [52].

A further concern is that a spontaneously broken discrete symmetry may lead to the formation of domain walls in the early Universe which could dominate the present energy density of the Universe [53-55]. For the case of gravity mediation, the domain walls would be expected to form around the SUSY-breaking scale $T \sim 10^{12} \mathrm{GeV}$. However, if inflation persists to lower temperatures, then the domain walls may be inflated away. It is key to observe that many mechanisms of baryogenesis are consistent with inflation persisting down to temperatures of $T \sim 10^{6} \mathrm{GeV}$ [56].

\section{String instanton solution}

In string theory models, it is possible for superpotential terms to arise from nonperturbative instanton effects. These are particularly well suited for open strings in braneworld scenarios such as IIA and IIB string theory. Intriguing applications of stringy instanton effects include the generation of Majorana neutrino mass terms, generation of Yukawa couplings and generation of the $\mu$ term in the superpotential $[57,58]$. In some D-brane models which include the MSSM at low energy, the superpotential $\mu$ term may be forbidden by $U(1)$ symmetries but then it is generated nonperturbatively via nongauge $D$-brane instanton effects. In this case, a $\mu$ term of the form

$$
W \sim \exp \left(-S_{\mathrm{cl}}\right) M_{s} H_{u} H_{d}
$$

can be induced where then $\mu \simeq \exp \left(-S_{\mathrm{cl}}\right) M_{s}$ and $M_{s}$ is the string mass scale. The exponential suppression leads to the possibility of a $\mu$ term far below the string scale. Of course, in this case one might expect the $\mu$ term to arise at any arbitrary mass scale below the string scale rather than fortuitously at the weak scale. If the $\mu$ term does arise at the weak scale from stringy instanton effects, then that value may act as an attractor such that soft terms like $m_{H_{u}}^{2}$ are pulled statistically to large values by the string theory landscape, but not so large that EW symmetry does not break. Then the weak scale value of $m_{H_{u}}^{2}$ is of comparable (negative) magnitude to $\mu$ (the naturalness condition) to ensure a universe with anthropically required electroweak symmetry breaking [19].

\section{6. $\mu$ solution in the $G_{2} \mathrm{MSSM}$}

In Ref. [59], the authors considered 11-dimensional $M$-theory compactified on a manifold of $G_{2}$ holonomy, and derived various phenomenological implications. They considered fields living in multiplets of $S U(5)$ so the doublet-triplet splitting problem is present. As opposed to string theory models compactified on orbifolds, in $M$-theory the matter fields live only in four dimensions so a different solution to the $\mu$ problem is required. Witten suggested the existence of an additional discrete symmetry which forbids the $\mu$ term from appearing but which allows the Higgs triplets to gain large enough masses so as to evade proton decay constraints [60]. In Ref. [61], it was shown that a $\mathbb{Z}_{4}$ symmetry is sufficient to forbid the $\mu$ term and other dangerous $R$-parity-violating (RPV) operators while allowing massive Higgs triplets. The $\mathbb{Z}_{4}$ discrete symmetry is assumed to be broken via moduli stabilization so that a small $\mu$ term develops.

In the $G_{2}$ MSSM, the gravitino gains mass from nonperturbative effects (such as gaugino condensation) in the hidden sector so that $m_{3 / 2} \sim \Lambda_{h}^{3} / m_{P}^{2} \sim 10-200 \mathrm{TeV}$. Matter scalar soft masses are expected at $m_{\phi} \sim m_{3 / 2}$ so they should be very heavy [and likely unnatural in the context of Eq. (1)]. In contrast, gauginos gain mass from the gauge kinetic function which depends on the VEVs of moduli fields so they are expected to be much lighter, $m_{\lambda} \sim \mathrm{TeV}$ scale and in fact these may have dominant anomaly-mediated SUSY breaking contributions [62] (with comparable moduli-mediated SUSY-breaking contributions) so that the wino may be the lightest of the gauginos. The dominant contribution to the $\mu$ parameter arises from Kähler contributions à la Giudice-Masiero and these are expected to be $\mu \sim c \frac{\left\langle S_{i}\right\rangle}{m_{p}} m_{3 / 2} \sim 0.1 m_{3 / 2}$ (where $c$ is some constant $\sim 1$ ) and thus is suppressed compared to scalar soft masses, but perhaps comparable to gaugino masses. ${ }^{4}$

\section{B. Extended MSSM-type solutions}

\section{NMSSM: Added singlet with $\mathbb{Z}_{3}$ discrete symmetry}

The case of adding an additional visible-sector gaugesinglet superfield $S$ to the MSSM leads to the next-tominimal SSM (NMSSM) [64]. Some motivation for the NMSSM can originate in string theory models such as

\footnotetext{
${ }^{4}$ While the $G_{2}$ MSSM $\mu$ solution is not related to solving the strong $C P$ problem, a discussion on solving the strong $C P$ problem within the $G_{2}$ MSSM can be found in Ref. [63].
} 
heterotic orbifolds where the $\mu$ term arises as an effective term from couplings of the Higgs pair to a singlet field [45]. Without imposing any symmetry to forbid singlet couplings, we can write a generic NMSSM superpotential as follows:

$$
\begin{aligned}
W_{\mathrm{NMSSM}}= & W_{\mathrm{MSSM}}(\mu=0)+\lambda_{\mu} S H_{u} H_{d}+\xi_{F} S+\frac{1}{2} \mu_{S} S^{2} \\
& +\frac{1}{3} \kappa S^{3}
\end{aligned}
$$

and the corresponding soft terms

$$
\begin{aligned}
\mathcal{L}_{\mathrm{soft}}^{\mathrm{NMSSM}}= & \mathcal{L}_{\mathrm{soft}}^{\mathrm{MSSM}}-\left(a_{\lambda} S H_{u} H_{d}+B \mu H_{u} H_{d}+\frac{1}{3} a_{\kappa} S^{3}\right. \\
& \left.+\frac{1}{2} b_{S} S^{2}+t S+\text { c.c. }\right)-m_{S}^{2}|S|^{2} .
\end{aligned}
$$

Here $W_{\text {MSSM }}(\mu=0)$ denotes the superpotential for the MSSM but without the $\mu$ term. The tadpole $t$ in Eq. (11) may have destabilizing quadratic divergences and must be suppressed [65]. A $\mathbb{Z}_{3}$ discrete symmetry is usually imposed wherein chiral superfields transform as $\phi \rightarrow$ $e^{2 \pi i / 3} \phi$ which sends the dimensionful couplings $\xi_{F}, \mu$, $\mu_{S}, B \mu, b_{S}$ and $t$ to zero (only cubic couplings are allowed) at the expense of possibly introducing domain walls into the early Universe after the electroweak phase transition [66]. (Some means of avoiding domain walls were proposed in Refs. [67].) By minimizing the scalar potential, now including the new singlet scalar $S$, the VEVs $v_{u}, v_{d}$ and $v_{s}$ are induced. An effective $\mu$ term emerges with

$$
\mu_{\mathrm{eff}}=\lambda_{\mu} v_{s} .
$$

An attractive alternative choice for a $\mu$-forbidding symmetry other than the (perhaps ad hoc) $\mathbb{Z}_{3}$ would be one of the anomaly-free discrete $R$ symmetries $\mathbb{Z}_{4}^{R}$ or $\mathbb{Z}_{8}^{R}$ [41]. Like the $\mathbb{Z}_{3}$ discrete symmetry, the $\mathbb{Z}_{8}^{R}$ symmetry also forbids the dangerous divergent tadpole term. The $\mathbb{Z}_{4}^{R}$ symmetry would allow the linear singlet term, but it can be argued that in the effective theory the linear term appears when the fields with which the singlet field is coupled acquire VEVs. If these fields belong to the hidden sector, then the coupling will be suppressed by some high mass scale ranging as high as $m_{P}$ in the case of gravity mediation. In this case the linear singlet term will be present but it will be highly suppressed [41].

Thus, all the advantages of the $\mathbb{Z}_{3}$ discrete symmetry can be obtained by imposing instead either a $\mathbb{Z}_{4}^{R}$ or $\mathbb{Z}_{8}^{R}$ symmetry: this then avoids the disadvantages (the ad hocness and introduction of domain walls into the early Universe after the electroweak phase transition) inherent in the $\mathbb{Z}_{3}$ discrete symmetry.

The added singlet superfield $S$ in the NMSSM leads to new scalar and pseudoscalar Higgs fields which can mix with the usual MSSM Higgses for $v_{s} \sim v_{u, d}$. So far, LHC Higgs coupling measurements favor an SM-like Higgs so one might expect $v_{s} \gg v_{u, d}$ which may lead one to an unnatural value of $\mu_{\text {eff }}$. The superfield $S$ also contains a spin- $\frac{1}{2}$ singlino $\tilde{s}$ which may mix with the usual neutralinos and might even be the LSP [68]. In the NMSSM, an additional Higgs quartic potential term is generated from the $F$ term of the singlet superfield, and thus the SM-like Higgs mass $125 \mathrm{GeV}$ is explained more easily without introducing large one-loop corrections. This feature can make the NMSSM more attractive to those who are uncomfortable with an MSSM Higgs of mass $m_{h} \simeq 125 \mathrm{GeV}$ [69].

\section{2. $n M S S M$}

An alternative singlet extension of the MSSM is the nearly minimal supersymmetric standard model (nMSSM) (also sometimes called the minimal nonminimal supersymmetric standard model) [70,71]. The nMSSM, like the NMSSM, solves the $\mu$ problem via an added singlet superfield $S$. But in the nMSSM, the model is founded on a discrete $R$ symmetry-either $\mathbb{Z}_{5}^{R}$ or $\mathbb{Z}_{7}^{R}$. Discrete $R$-charge assignments for $\mathbb{Z}_{5}^{R}$ are shown in Table II. The tree-level superpotential is given by

$$
\begin{aligned}
W_{\mathrm{nMSSM}} \ni & \lambda_{\mu} S H_{u} H_{d}+f_{u} Q H_{u} U^{c}+f_{d} Q H_{d} D^{c} \\
& +f_{\ell} L H_{d} E^{c}+f_{\nu} L H_{u} N^{c}+\frac{1}{2} M_{N} N^{c} N^{c}
\end{aligned}
$$

so that unlike the NMSSM with $\mathbb{Z}_{3}$ symmetry, the $\kappa S^{3}$ term is now forbidden. This is why the model is touted as a more minimal extension of the MSSM. The discrete $R$ symmetry is broken by SUSY-breaking effects in gravity mediation. Then, in addition to the above terms, an effective potential tadpole contribution

$$
W_{\mathrm{nMSSM}}^{\mathrm{tad}} \ni \xi_{F} S
$$

is induced at the six-loop level or higher where $\xi_{F} \sim m_{3 / 2}^{2}$ (along with a corresponding soft SUSY-breaking term). Due to the lack of the discrete global $\mathbb{Z}_{3}$ symmetry, the nMSSM then avoids the domain-wall and weak-scale axion problems that might afflict the NMSSM.

Like the NMSSM, the nMSSM will include added scalar and pseudoscalar Higgs particles along with a fifth neutralino. However, due to the lack of the $S$ self-coupling term and the presence of the tadpole term, the mass eigenstates and couplings of the added matter states will differ from

TABLE II. Charge assignments for various superfields of the nMSSM with a $\mathbb{Z}_{5}^{R}$ discrete $R$ symmetry.

\begin{tabular}{lccccccccc}
\hline \hline Multiplet & $H_{u}$ & $H_{d}$ & $Q_{i}$ & $U_{i}^{c}$ & $D_{i}^{c}$ & $L_{i}$ & $E_{i}^{c}$ & $N^{c}$ & $S$ \\
\hline $\mathbb{Z}_{5}^{R}$ & 2 & 2 & 4 & 6 & 6 & 4 & 6 & 6 & 3 \\
\hline \hline
\end{tabular}


the NMSSM [72-76]. The neutralino in the nMSSM is very light, mostly below $50 \mathrm{GeV}$, but it is hard to get lower than $30 \mathrm{GeV}$ due to the dark matter relic density constraint. ${ }^{5}$ Since the neutralinos are so light it is very likely that a chargino will decay into either an MSSM-like $\chi_{2}^{0}$ or a singlino $\chi_{1}^{0}$, giving rise to a five-lepton final state. A further decay of the neutralino can give rise to a seven-lepton state. These kinds of multilepton events are more likely in the nMSSM than in the NMSSM. Also, since in the nMSSM the neutralino can be so light, deviations in Higgs boson $h$ decay branching fractions become more likely than in the case of the NMSSM [74,75].

\section{3. $\mu$-from- $\nu S S M$}

The $\mu$-from- $\nu \mathrm{SSM}$ ( $\mu \nu \mathrm{SSM})$ [78] is in a sense a more minimal version of the NMSSM in that it makes use of the gauge-singlet right-hand-neutrino superfields $N_{i}^{c}$ to generate a $\mu$ term. The $\mu \nu \mathrm{SSM}$ first requires a $\mathbb{Z}_{3}$ symmetry to forbid the usual $\mu$ term (and also a usual Majorana neutrino mass term $M_{i} N^{c} N^{c}$ ). The superpotential is given by

$$
\begin{aligned}
W \ni & f_{u} Q H_{u} U^{c}+f_{d} Q H_{d} D^{c}+f_{\ell} L H_{d} E^{c}+f_{\nu} L H_{u} N^{c} \\
& +\lambda_{\mu i} N_{i}^{c} H_{u} H_{d}+\frac{1}{3} \kappa_{i j k} N_{i}^{c} N_{j}^{c} N_{k}^{c} .
\end{aligned}
$$

If the scalar component of one of the right-hand-neutrino superfields $\tilde{\nu}_{R i}$ of $N_{i}^{c}$ gains a weak scale VEV, then an effective $\mu$ term develops,

$$
\mu_{\text {eff }}=\lambda_{\mu i}\left\langle\tilde{\nu}_{R i}\right\rangle
$$

along with a weak scale Majorana neutrino mass term $M_{N j k} \sim \kappa_{i j k}\left\langle\tilde{\nu}_{R i}\right\rangle$. By taking small enough neutrino Yukawa couplings, a weak-scale seesaw develops which can accommodate the measured neutrino masses and mixings.

The $\mu \nu \mathrm{SSM}$ develops bilinear $R$-parity-violating terms via the superpotential $f_{\nu} L H_{u} N^{c}$ term so that the lightest $\mu \nu \mathrm{SSM}$ particle is not stable and does not comprise dark matter: $\tilde{\chi}_{1}^{0} \rightarrow W^{(*)} \ell$ and other modes. As an alternative, a gravitino LSP is suggested with an age longer than the age of the Universe: it could decay as $\tilde{G} \rightarrow \nu \gamma$ and possibly yield gamma-ray signals from the sky [79]. The phenomenology of the $\mu \nu \mathrm{SSM}$ also becomes more complex: now the neutrinos inhabit the same mass matrix as neutralinos, leptons join charginos in another mass matrix and Higgs scalars and sneutrinos inhabit a third mass matrix (albeit with typically small mixing effects). Collider signals are strongly modified from usual MSSM expectations [80].

While the $\mu \nu \mathrm{SSM}$ may be considered the most minimal model to solve the $\mu$ problem, it suffers the same $\mathbb{Z}_{3}$ domain-wall problem as the NMSSM (and perhaps the same routes to avoidance [67]). Also, in the context of

\footnotetext{
${ }^{5}$ Radiative corrections can change this result: see Ref. [77].
}

TABLE III. Charge assignments for various superfields of a $U(1)^{\prime}$ model $[83,84]$.

\begin{tabular}{lcccccccr}
\hline \hline Multiplet & $H_{u}$ & $H_{d}$ & $Q_{i}$ & $U_{i}^{c}$ & $D_{i}^{c}$ & $L_{i}$ & $E_{i}^{c}$ & $S$ \\
\hline$(2 \sqrt{10}) Q^{\prime}$ & -2 & -3 & 1 & 1 & 2 & 2 & 1 & 5 \\
\hline \hline
\end{tabular}

GUTs, the role that the $N_{i}^{c}$ field plays in the 16-dimensional spinor of $S O(10)$ would have to be abandoned.

\section{C. $\boldsymbol{\mu}$ from an extra local $U(1)^{\prime}$}

In this class of models [81-85], an SM singlet superfield $S$ is introduced which is charged under a new $U(1)^{\prime}$ gauge interaction, so terms with mass dimensions in Eq. (10) are forbidden. Due to the $U(1)^{\prime}$ gauge charges of $S$, the cubic coupling $S^{3}$ is also absent. We will see below three representative realizations of this class of model.

\section{CDEEL model}

Cvetic, Demir, Espinosa, Everett, and Langacker (CDEEL) [81] proposed a $U(1)^{\prime}$-extended gauge symmetry model as emblematic of fermionic orbifold string compactifications. While the usual $\mu$ term is forbidden by the extended gauge symmetry, the superpotential term

$$
W \ni \lambda_{\mu} S H_{u} H_{d}
$$

is allowed and under $U(1)^{\prime}$ breaking $S$ develops a $\operatorname{VEV}\langle S\rangle \sim$ $m_{\text {weak }}$ such that a $\mu$ term is generated $\mu_{\text {eff }}=\lambda_{\mu}\langle S\rangle$ along with an additional weak-scale $Z^{\prime}$ gauge boson. Forbidding the $\mu$ term via a gauge symmetry avoids the gravity spoliation/ global symmetry problem. In addition, the $\mu$ term is linked to EW symmetry breaking and this would be expected to occur at $m_{\text {weak }}$ rather than $m_{\text {soft }}$. The $U(1)^{\prime}$ breaking can occur either via large soft SUSY-breaking trilinear couplings or via radiative corrections driving certain mass-squared terms negative. A way to test this class of models, in the exotica decoupling limit, is to search for new $Z^{\prime}$ gauge bosons with exotic decays to light Higgsinos [84].

To maintain anomaly cancellation, a variety of (intermediate scale) exotic quark and lepton fields must be introduced along with extra SM gauge singlets. If these new states come in GUT representations, then gauge coupling unification can be maintained. A set of possible $U(1)^{\prime}$ gauge charges are listed in Table III.

\section{2. sMSSM model}

An alternative $U(1)^{\prime}$-extended MSSM (abbreviated as sMSSM) [86,87] also solves the $\mu$ problem by invoking multiple SM singlet superfields charged under $U(1)^{\prime}$ symmetry. In this model, a visible-sector singlet field $S$ directly couples to Higgs doublets but avoids stringent constraints on having an additional weak scale $Z^{\prime}$ gauge boson by introducing as well a secluded sector containing 
three additional singlets $S_{1}, S_{2}, S_{3}$ charged under $U(1)^{\prime}$. The superpotential is given by

$$
W_{\text {sMSSM }} \ni \lambda_{\mu} S H_{u} H_{d}+\lambda_{s} S_{1} S_{2} S_{3}
$$

so that the secluded sector has a nearly $F$ - and $D$-flat scalar potential. The $U(1)^{\prime}$ and electroweak symmetry breaking then occurs as a result of SUSY-breaking $A$ terms. Then the secluded sector scalars can obtain VEVs much larger than the weak scale; if also the trilinear singlet coupling $\lambda_{s}$ is small, then the additional $Z^{\prime}$ essentially decouples. Nonetheless, additional Higgs bosons and singlinos appear in the weak-scale effective theory so that this model phenomenologically resembles the nMSSM (described in Sec. II B 2) which has very different manifestations from what is expected from the CDEEL $U(1)^{\prime}$ model.

\section{HPT model}

The Hundi-Pakvasa-Tata (HPT) model [82] also solves the SUSY $\mu$ problem by positing an additional $U(1)^{\prime}$ gauge symmetry in a supergravity context. The $U(1)^{\prime}$ charges of the multiplets in the HPT scheme are shown in Table IV. With these $U(1)^{\prime}$ charge assignments, the $\mu$ term is forbidden in the superpotential but (unlike the CDEEL model) a dimension-four term as the $\mu$ solution $a$ la the Kim-Nilles model is allowed:

$$
W \ni \lambda_{\mu} S^{2} H_{u} H_{d} / M_{p}
$$

The $U(1)^{\prime}$ gauge symmetry also forbids trilinear RPV couplings and dangerous $p$-decay operators. When the $U(1)^{\prime}$ breaks (at an intermediate scale $Q \sim 10^{11} \mathrm{GeV}$ ), the $S$ field acquires a VEV to yield an effective $\mu$ parameter of the required magnitude.

A distinctive feature of the HPT model is that a bilinear RPV (bRPV) term, $L H_{u}$ is allowed at the right magnitude so as to generate phenomenologically allowed neutrino masses [88]. The desired pattern of neutrino masses and mixing angles are also accommodated through radiative corrections. The bRPV leads to an unstable lightest neutralino which decays via $\tilde{\chi}_{1}^{0} \rightarrow \ell W^{(*)}$ or $\nu Z^{(*)}$ and may lead to displaced vertices in collider events. Dark matter must be comprised of some other particles (e.g., axions). Also, the $U(1)^{\prime}$ is broken at the intermediate scale $Q \sim 10^{11} \mathrm{GeV}$ so that the additional $Z^{\prime}$ has a mass far beyond the reach of any collider.

Since solving the $\mu$ problem as well as generating the neutrino mass scale of suitable order requires the introduction of a new gauge group $U(1)^{\prime}$, care must be taken so that

TABLE IV. Charge assignments for various superfields of the HPT $U(1)^{\prime}$ supergravity model [82].

\begin{tabular}{lcccccccr}
\hline \hline Multiplet & $H_{u}$ & $H_{d}$ & $Q_{i}$ & $U_{i}^{c}$ & $D_{i}^{c}$ & $L_{i}$ & $E_{i}^{c}$ & $S$ \\
\hline$Q^{\prime}$ & 25 & -31 & 0 & -25 & 31 & 2 & 29 & 3 \\
\hline \hline
\end{tabular}

associated anomalies are canceled. Anomaly cancellation requires introducing various additional exotic fields including color-triplet $K_{i}$ and $K_{i}^{\prime}$ states. The lightest of these leads to stable weak-scale exotic hadrons which may also yield highly ionizing tracks at collider experiments. In the HPT scheme, gauge coupling unification may be upset.

\section{Solutions related to Peccei-Quinn symmetry breaking}

In this subsection, we examine natural $\mu$-term solutions related to the PQ symmetry used to solve the strong $C P$ problem. In this class of models, the $\mu$ term is forbidden by the PQ symmetry, but generated once the PQ symmetry is spontaneously broken. Then the model also provides a solution to the strong $C P$ problem and generates axion dark matter. In Secs. II D 1, II D 2, and II D 3, we review $\mu$-term generation models with various sources of PQ breaking.

Meanwhile, imposing a global symmetry causes the "quality" issues of the symmetry which may spoil the PQ solution to the strong $C P$ problem, since global symmetries are not protected from quantum gravity effects. In Sec. II D 4 , we discuss a criterion for protecting the PQ solution to the strong $C P$ problem, and in Sec. II D 5 we present examples based on discrete $R$ symmetries which satisfy the gravitysafety criterion and can be considered as generating an accidental, approximate PQ symmetry. Also, we review the natural Higgs-flavor-democracy solution which contains an approximate PQ symmetry from a discrete symmetry in Sec. II D 6.

Finally, we review $\mu$-term generation by the breaking of PQ symmetry from SUSY breaking: radiative breaking of PQ symmetry (Sec. II D 7), breaking of an accidental approximate PQ symmetry from a gauged $U(1)_{R}$ symmetry (Sec. II D 8) and a $\mathbb{Z}_{22}$ discrete gauge symmetry (Sec. II D 9) by a large negative trilinear term.

\section{Kim-Nilles solution}

Kim and Nilles (KN) [29] presented the first formulation of the SUSY $\mu$ problem along with a proposed solution. In Ref. [29], it was proposed that there exists a global PQ symmetry $U(1)_{\mathrm{PQ}}$ which is needed at first as a solution to the strong $C P$ problem. The PQ symmetry is implemented in the context of the supersymmetrized version of the DFSZ [89] axion model ${ }^{6}$ wherein the Higgs multiplets carry PQ charges e.g., $Q_{\mathrm{PQ}}\left(H_{u}\right)=Q_{\mathrm{PQ}}\left(H_{d}\right)=-1$ so that the $\mu$ term is forbidden by the global $U(1)_{\mathrm{PQ}}$. Next, the Higgs multiplets are coupled via a nonrenormalizable interaction to a SM gauge-singlet field $X$ which carries a PQ charge $Q_{\mathrm{PQ}}(X)=+2 /(n+1)$ :

\footnotetext{
${ }^{6}$ In the DFSZ axion model [89], the SM is extended to include two Higgs doublets which then couple to singlets which contain the axion.
} 
TABLE V. PQ charge assignments for various superfields of the $\mathrm{KN}$ model with $n=1$. One may add multiples of weak hypercharge or $B-L$ to these so their values are not unique.

\begin{tabular}{lcccccccccc}
\hline \hline Multiplet & $H_{u}$ & $H_{d}$ & $Q_{i}$ & $L_{i}$ & $U_{i}^{c}$ & $D_{i}^{c}$ & $E_{i}^{c}$ & $\mathrm{X}$ & $\mathrm{Y}$ & $\mathrm{Z}$ \\
\hline PQ charge & -1 & -1 & +1 & +1 & 0 & 0 & 0 & +1 & -1 & 0 \\
\hline \hline
\end{tabular}

$$
W_{\mu} \ni \frac{\lambda_{\mu}}{m_{P}^{n}} X^{n+1} H_{u} H_{d}
$$

for $n \geq 1$.

It is arranged to spontaneously break PQ symmetry by giving the $X$ field a VEV $\langle X\rangle$ which also generates a (nearly) massless axion $a$ which solves the strong $C P$ problem. To obtain cosmologically viable axions-with $\langle X\rangle \sim 10^{11} \mathrm{GeV}$ and with $m_{p} \simeq 2.4 \times 10^{18} \mathrm{GeV}$-we can obtain the $\mu$ parameter of the order of $m_{3 / 2}$ only if $n=1$ [for which $Q_{\mathrm{PQ}}(X)=+1$ ]. The matter superfields also carry appropriate PQ charge so as to allow the MSSM trilinear superpotential terms: see Table V.

The intermediate PQ breaking scale can be gained from a PQ superpotential of the form

$$
W_{\mathrm{PQ}}=\lambda_{\mathrm{PQ}} Z\left(X Y-v_{\mathrm{PQ}}^{2}\right) .
$$

The scalar components of $X$ and $Y$ develop VEVs $\langle X\rangle=$ $\langle Y\rangle=v_{\mathrm{PQ}}$ such that a $\mu$ term is generated:

$$
\mu=\lambda_{\mu}\langle X\rangle^{2} / m_{P}
$$

This value of the $\mu$ term $\mu \sim \lambda_{\mu} v_{\mathrm{PQ}}^{2} / m_{P}$ is to be compared to the soft breaking scale in models of gravity mediation: $m_{\text {soft }} \sim m_{3 / 2} \sim m_{\text {hidden }}^{2} / m_{P}$. Here, $v_{\mathrm{PQ}}$ is identified as $v_{\mathrm{PQ}} \sim$ $m_{\text {hidden }}$ and thus $\mu$ is obtained as $\mu \sim m_{3 / 2}$. But, a value $\mu \sim m_{\text {weak }} \ll m_{\text {soft }} \sim m_{3 / 2}$ can be accommodated for $v_{\mathrm{PQ}}<m_{\text {hidden }}$, i.e., if the scale of PQ breaking lies somewhat below the mass scale associated with hidden-sector SUSY breaking. ${ }^{7,8}$ A virtue of the $\mathrm{KN}$ solution is that it combines a solution to the strong $C P$ problem with a solution to the SUSY $\mu$ problem which also allows for a little hierarchy. A further benefit is that it provides an additional dark matter particle-namely the DFSZ [89] axion-to coexist with the (thermally under-produced) Higgsino-like weakly interacting massive particle (WIMP)

\footnotetext{
${ }^{7}$ In models with SUSY breaking arising from e.g., gaugino condensation at an intermediate scale $\Lambda_{h}, m_{3 / 2} \sim \Lambda_{h}^{3} / m_{P}^{2}$ in which case we would define $m_{\text {hidden }}^{2} \sim \Lambda_{h}^{3} / m_{p}$.

${ }^{8}$ The model of Ref. [90] describes a more complete ultraviolet theory which includes a mechanism to get $v_{\mathrm{PQ}}$ in the intermediate scale through the introduction of a chiral superfield in the hidden brane, yielding an ultraviolet-suppressed term in the hidden brane which gives rise to $\mu \sim m_{\text {weak }}$ when SUSY is broken in the hidden brane through the shining mechanism [91].
}

from natural SUSY. Thus, dark matter is then expected to be comprised of a WIMP/axion admixture [92,93]. For the lower range of the PQ scale $v_{\mathrm{PQ}}$, the dark matter tends to be axion dominated with typically 10-20\% WIMPs by mass density [94]. For larger $v_{\mathrm{PQ}}$ values, nonthermal processes such as saxion and axino [95] decay augment the WIMP abundance while for even larger values of $v_{\mathrm{PQ}}$ the Higgsinolike WIMPs are overproduced and one typically runs into big bang nucleosynthesis constraints from late-decaying neutral particles (saxions and axinos) or overproduction of relativistic axions from saxion decay which contribute to the effective number of neutrino species $N_{\text {eff }}$ (which is found to be $N_{\text {eff }}=3.13 \pm 0.32$ from the recent Particle Data Group tabulation [96]). In the context of the DFSZ model embedded within the MSSM, the presence of Higgsinos in the $a \gamma \gamma$ triangle diagram is expected to reduce the axion-photonphoton coupling to levels below present sensitivity making the SUSY DFSZ axion very challenging to detect [97].

\section{Chun-Kim-Nilles model}

In the Chun-Kim-Nilles (CKN) model [98], it is assumed that SUSY is broken in the hidden sector due to gaugino condensation $\langle\lambda \lambda\rangle \sim \Lambda_{h}^{3} \sim\left(10^{13} \mathrm{GeV}\right)^{3}$ in the presence of a hidden $S U(N)_{h}$ gauge group. Furthermore, there may be vector-like hidden-sector quark chiral superfields present $Q$ and $Q^{c}$ which transform as $N$ and $N^{*}$ under $S U(N)_{h}$. The Higgs and hidden quark superfields carry PQ charges as in Table VI: this allows for the presence of a superpotential term

$$
W_{\mathrm{CKN}} \ni \frac{\lambda_{\mu}}{m_{P}} Q Q^{c} H_{u} H_{d}
$$

Along with gauginos condensing at a scale $\Lambda_{h}$ to break SUGRA with $m_{3 / 2} \sim \Lambda_{h}^{3} / m_{P}^{2}$, the hidden-sector scalar squarks condense at a scale $\Lambda<\Lambda_{h}$ to break the PQ symmetry and to generate a $\mu$ term

$$
\mu_{\mathrm{eff}} \sim \lambda_{\mu} \Lambda^{2} / m_{P}
$$

Thus, this model provides a framework for $\mu<m_{\text {soft }}$. It also generates a DFSZ axion to solve the strong $C P$ problem along with a string model-independent (MI) axion which could provide a quintessence solution for the cosmological constant (CC) [99]. The CC arises from the very low-mass MI axion field slowly settling to the minimum of its potential.

TABLE VI. PQ charge assignments for various superfields of the $\mathrm{CKN}$ model.

\begin{tabular}{lccccccc}
\hline \hline Multiplet & $H_{u}$ & $H_{d}$ & $Q$ & $Q^{c}$ & $Q_{i}$ & $U_{i}^{c}$ & $D_{i}^{c}$ \\
\hline PQ charge & -1 & -1 & 1 & 1 & 0 & 1 & 1 \\
\hline \hline
\end{tabular}




\section{BASTERO-GIL-KING/EYTON-WILLIAMS-KING solution linked to inflation and strong $C P$}

In Refs. [100,101], a model was proposed with the superpotential

$$
W_{\mathrm{EWK}} \ni \lambda_{\mu} \phi H_{u} H_{d}+\kappa \phi N^{2}
$$

where the $\phi$ field plays the role of the inflaton and the $N$ field is a waterfall field leading to hybrid inflation in the early Universe [102]. Although the model appears similar to the NMSSM, it is based on a PQ rather than $\mathbb{Z}_{3}$ symmetry with charges as in Table VII. Thus, it avoids the NMSSM domain-wall problems which arise from a postulated global $\mathbb{Z}_{3}$ symmetry. By augmenting the scalar potential with soft breaking terms, the $\phi$ and $N$ fields gain VEVs of order some intermediate scale $Q \sim 10^{12} \mathrm{GeV}$ so that Yukawa couplings $\lambda_{\mu}$ and $\kappa$ are of order $10^{-10}$. Such tiny Yukawa couplings might arise from type-I string theory constructs [103]. To fulfill the inflationary slow-roll conditions, the field $\phi$ must gain a mass of less than $5-10 \mathrm{MeV}$ and a reheat temperature of $1-10 \mathrm{GeV}$. Domain walls from breaking of the PQ symmetry are inflated away.

\section{Global symmetries and gravity}

It is well known that gravitational effects violate global symmetries, as has been considered via black hole "no-hair" theorems [34] and wormhole effects [35]. In such cases, it has been questioned whether the PQ mechanism can be realistic once one includes gravity or embeds the SUSY PQ theory into a UV-complete string framework [36-38,104,105]. Indeed, Kamionkowski and MarchRussell (KMR) [38] considered the effect of gravitational operators such as

$$
V(\phi) \ni \frac{g}{m_{P}^{2 m+n-4}}|\phi|^{2 m} \phi^{n}+\text { H.c. }+c
$$

involving PQ charged fields $\phi$ in the scalar potential upon the axion potential. In the case of $2 m+n=5$, i.e., a term suppressed by a single power of $m_{P}$, these gravitational terms would displace the minimum of the PQ axion potential such that the QCD $C P$-violating term $G_{\mu \nu A} \tilde{G}_{A}^{\mu \nu}$ settles to a nonzero minimum thus destroying the PQ solution to the strong $C P$ problem. To maintain $\bar{\theta} \lesssim 10^{-10}$, KMR calculated that all gravitational operators contributing to the axion potential should be

TABLE VII. PQ charge assignments for various superfields of the EWK model.

\begin{tabular}{lcccc}
\hline \hline Multiplet & $H_{u}$ & $H_{d}$ & $\phi$ & $N$ \\
\hline PQ charge & -1 & -1 & +2 & -1 \\
\hline \hline
\end{tabular}

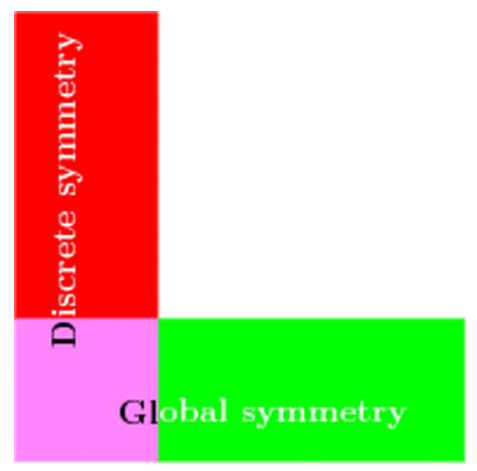

FIG. 1. Kim diagram [108,109] where the column represents an infinite sequence of Lagrangian terms obeying gravity-safe discrete symmetry while the row represents an infinite sequence of terms obeying the global symmetry. The green-region terms are gravity unsafe while the red region violates the global symmetry. The lavender terms are gravity safe and obey the global symmetry.

suppressed by at least powers of $\left(1 / m_{P}\right)^{8}$. This is indeed a formidable constraint!

To avoid such terms, additional symmetries are required [106]. In string theory, it is known that discrete symmetries arising from gauge symmetries are gravity safe, as are other discrete symmetries or $R$ symmetries arising from string compactification. ${ }^{9}$ In Fig. 1 the Kim diagram is shown $[108,109]$. The red/lavender column denotes an infinite set of Lagrangian terms in the model under consideration which obey some exact, gravity-safe, discrete symmetry. Of this set of terms, the few lower-order terms, denoted by the lavender region, obey an exact global symmetry, understood here to be the PQ symmetry whose breaking yields the QCD axion. The red-shaded terms obey the discrete symmetry but violate any global symmetry. The green/lavender row denotes the full, infinite set of global symmetry terms, of which the green-shaded terms are not gravity safe. If the discrete symmetry is strong enough, then the gravity-unsafe terms will be sufficiently suppressed. The global PQ symmetry is expected to be approximate. The question then is: is it sufficiently strong so as to be gravity safe? Some additional gravity-safe symmetry is required to ensure that the PQ mechanism is robust. The lavender region represents gravity-safe terms which obey the global symmetry.

\section{Gravity-safe symmetries: gauge symmetries or $\boldsymbol{R}$ symmetries, continuous or discrete}

Given that global symmetries are not gravity safe (and hence not fundamental), it is common to turn to gauge symmetries as a means to forbid the $\mu$ term. Some models based on an extra local $U(1)^{\prime}$ were examined in Sec. II C. Some problems with this approach emerge in that one has

\footnotetext{
${ }^{9}$ See e.g., Ref. [107].
} 
to suitably hide any new gauge bosons associated with the extra gauge symmetry and one must also typically introduce (and hide) extra exotic matter which may be needed to ensure anomaly cancellation. In addition, such exotic matter may destroy the desirable feature of gauge coupling unification should the new exotica not appear in complete GUT multiplets.

An alternative approach is to introduce discrete gauge symmetries [106,110]. Such $\mathbb{Z}_{M}$ symmetries may emerge from a local $U(1)^{\prime}$ when a charge $M$ object [charged under the new $\left.U(1)^{\prime}\right]$ condenses at very high energy leaving a discrete $\mathbb{Z}_{M}$ gauge symmetry in the low-energy effective theory. Since the $\mathbb{Z}_{M}$ emerges from a local gauge theory, it remains gravity safe. In Sec. II D 9, the MBGW model [39] which is based on a $\mathbb{Z}_{22}$ discrete gauge symmetry is examined. The model under $\mathbb{Z}_{22}$ is found to be anomaly free and is used to not only forbid the $\mu$ term but also generate a PQ symmetry needed to solve the strong $C P$ problem. The lowest-order PQ-violating term allowed by the $\mathbb{Z}_{22}$ is sufficiently suppressed so that PQ arises as an accidental approximate global symmetry thereby rendering the model gravity safe. The $\mathbb{Z}_{22}$ discrete gauge charges of the multiplets turn out to be inconsistent with GUTs which should be manifested at some level in the high-energy theory. Also, the presence of a charge 22 object which condenses at some high energy scale may not be very plausible and might be inconsistent with the UV completion of the theory (i.e., lie in the swampland).

Continuous or discrete $R$ symmetries offer a further choice for gravity-safe symmetries. A solution using a continuous $U(1)_{R}$ symmetry was examined in Sec. II A 3. ${ }^{10}$ In the interest of minimality, it is noted that continuous $R$ symmetries are not consistent with the particle content of just the MSSM [112]. Then it is also of interest to examine the possibility of discrete remnant $R$ symmetries $\mathbb{Z}_{N}^{R}$ which arise upon compactification of the full Lorentz symmetry of ten-dimensional string theories. $R$ symmetries are characterized by the fact that superspace coordinates $\theta$ carry nontrivial $R$ charge: in the simplest case, $Q_{R}(\theta)=+1$ so that $Q_{R}\left(d^{2} \theta\right)=-2$. For the Lagrangian $\mathcal{L} \ni \int d^{2} \theta W$ to be invariant under $\mathbb{Z}_{N}^{R}$ symmetry, the superpotential $W$ must carry $Q_{R}(W)=2+$ integer multiples of $N$.

These remnant discrete $R$ symmetries $\mathbb{Z}_{N}^{R}$-if sufficiently strong — can forbid lower-order operators in powers of $1 / m_{P}$ which would violate putative global symmetries such as PQ. Such a built-in mechanism from string theory may enable the PQ symmetry to be strong enough to support the axion solution to the strong $C P$ problem. Since the $R$ symmetry is necessarily supersymmetric (it acts on superspace coordinates), this is another instance of how the implementation of the axion solution to the strong $C P$ problem is enhanced and made more plausible by the presence of supersymmetry. However, not all possible

\footnotetext{
${ }^{10}$ See also Ref. [111].
}

TABLE VIII. Derived MSSM field $R$-charge assignments for various anomaly-free discrete $\mathbb{Z}_{N}^{R}$ symmetries which are consistent with $S U(5)$ or $S O(10)$ unification (from Ref. [41]).

\begin{tabular}{lccccr}
\hline \hline Multiplet & $\mathbb{Z}_{4}^{R}$ & $\mathbb{Z}_{6}^{R}$ & $\mathbb{Z}_{8}^{R}$ & $\mathbb{Z}_{12}^{R}$ & $\mathbb{Z}_{24}^{R}$ \\
\hline$H_{u}$ & 0 & 4 & 0 & 4 & 16 \\
$H_{d}$ & 0 & 0 & 4 & 0 & 12 \\
$Q$ & 1 & 5 & 1 & 5 & 5 \\
$U^{c}$ & 1 & 5 & 1 & 5 & 5 \\
$E^{c}$ & 1 & 5 & 1 & 5 & 5 \\
$L$ & 1 & 3 & 5 & 9 & 9 \\
$D^{c}$ & 1 & 3 & 5 & 9 & 9 \\
$N^{c}$ & 1 & 1 & 5 & 1 & 1 \\
\hline \hline
\end{tabular}

$R$ symmetries are suitable candidates for a fundamental symmetry. Table VIII (as derived in Refs. [40,41]) shows the $R$ symmetries along with the $R$ charges of the multiplets which are consistent with either $S U(5)$ or $S O(10)$ unification, are anomaly free (allowing for a Green-Schwarz term), forbid the $\mu$ term and also forbid the $R$-parityviolating and dimension-five proton decay operators and hence can serve the purpose of being a fundamental symmetry. In fact, the $\mathbb{Z}_{N}^{R}$ symmetries of Table VIII have been shown to be the only anomaly-free symmetries which allow for fermion masses and suppress the $\mu$ term while maintaining consistency with GUTs. As a bonus, they allow for neutrino masses while forbidding $R$ parity and dangerous proton decay operators. Implementation of the discrete $R$ symmetries is only possible in extra-dimensional GUTs, making their implementation in string compactifications very natural [113].

\section{Natural Higgs-flavor-democracy solution to the $\mu$ problem}

In Ref. [114], the $\mu$ problem was solved by introducing additional identical Higgs doublet superfields to those of the MSSM. The theory then contains a direct product of discrete interchange symmetries $S_{2}\left(H_{u}\right) \times S_{2}\left(H_{d}\right)$. This is Higgs-flavor democracy (HFD). Besides solving the $\mu$ problem, this mechanism also gives rise to an approximate PQ symmetry and hence a light QCD axion, thereby solving the strong $C P$ problem while avoiding the gravity spoliation problem. The HFD discrete symmetry can be found in several string theory models.

HFD: One starts by introducing two pairs of Higgs doublets at the GUT scale $m_{G}$, namely, $\left\{H_{u}^{(1)}, H_{d}^{(1)}\right\}$ and $\left\{H_{u}^{(2)}, H_{d}^{(2)}\right\}$. However, the weak-scale MSSM requires only one pair of Higgs doublets: $\left\{H_{u}, H_{d}\right\}$. If, at the GUT scale, the two pairs of Higgs doublets $H_{u}=$ $\left\{H_{u}^{(1)}, H_{u}^{(2)}\right\}$ and $H_{d}=\left\{H_{d}^{(1)}, H_{d}^{(2)}\right\}$ are indistinguishable then there must exist the permutation symmetries $S_{2}\left(H_{u}\right) \times S_{2}\left(H_{d}\right)$. Then the Higgsino mass matrix has a democratic form given by 


$$
\left\{\begin{array}{ll}
m_{G} / 2 & m_{G} / 2 \\
m_{G} / 2 & m_{G} / 2
\end{array}\right\}
$$

The Higgs mass eigenvalues are $m_{G}$ and 0. Hence, the Higgs pair in the weak-scale MSSM is found to be massless. Still, the model construction of the MSSM requires a massive Higgs pair at the weak scale with mass value $\mu$. In order to fulfill this criteria, the HFD must be broken and this mechanism results in $\mu \approx \mathcal{O}(\mathrm{TeV})$.

Generation of $\mu$ : The minimal Kahler potential is considered as $K=\Phi_{i} \Phi_{i}^{\dagger}+X_{i} X_{i}^{\dagger}+\bar{X}_{i} \bar{X}_{i}^{\dagger}$ where $\Phi_{i}$ $(i=1,2)$ is a doublet under the gauge group such as the Higgs superfield and $X_{i}$ and $\bar{X}_{i}(i=1,2)$ are singlets under the gauge group. Both $\Phi_{i}$ and $X_{i}$ and the corresponding barred fields obey the $S_{2} \times S_{2}$ symmetry. $X^{(0)}$ and $\bar{X}^{(0)}$ are SM singlet fields containing a very light QCD axion for $10^{9} \mathrm{GeV} \leq v_{\mathrm{PQ}} \leq 10^{12} \mathrm{GeV}$. With this construction, the $S_{2}(L) \times S_{2}(R)$-symmetric nonrenormalizable term is

$$
\begin{aligned}
W^{(\text {nonrenormalizable })}= & \sum_{i, j=1,2}\left(\frac{X^{(i)} \bar{X}^{(j)}}{m_{P}}\right) H_{u}^{(i)} H_{d}^{(j)} \\
& +\sum_{i j} \sum_{k l}\left(\frac{X^{(i)} \bar{X}^{(j)}}{m_{P}}\right) H_{u}^{(k)} H_{d}^{(l)} .
\end{aligned}
$$

With the HFD-breaking minimum at $\left\langle X_{1}\right\rangle=\left\langle\bar{X}_{1}\right\rangle=v_{\mathrm{PQ}}$ and $\left\langle X_{2}\right\rangle=\left\langle\bar{X}_{2}\right\rangle=0$, Eq. (26) becomes

$W^{(\text {nonrenormalizable })}=\frac{\lambda_{\mu} v_{\mathrm{PQ}}^{2}}{2 m_{P}}\left(H_{u}^{(0)}+H_{u}^{\left(M_{G}\right)}\right)\left(H_{d}^{(0)}+H_{d}^{\left(M_{G}\right)}\right)$.

This choice of HFD-breaking minimum is spontaneous. Thus we obtain $\mu=\frac{\lambda_{\mu} v_{\mathrm{PQ}}^{2}}{2 m_{P}}$. With $10^{10} \mathrm{GeV} \leq v_{\mathrm{PQ}} \leq 10^{12} \mathrm{GeV}$ and $\lambda_{\mu} \approx \mathcal{O}(1)$, we obtain $\mu \approx \mathcal{O}\left(0.1-10^{3} \mathrm{TeV}\right)$. The little hierarchy (LH) can be accommodated for the lower range of $v_{\mathrm{PQ}}$ or if $\lambda_{\mu}<1$.

Light $Q C D$ axion: Integrating out the heavy fields in Eq. (27), one obtains

$$
W=\frac{\lambda_{\mu} X^{(0)} \bar{X}^{(0)}}{2 m_{P}} H_{u}^{(0)} H_{d}^{(0)}
$$

The PQ charges of Higgs multiplets are obtained from their interaction with the quarks and the PQ charges of $X^{(0)}$ and $\bar{X}^{(0)}$ are defined by Eq. (28). Thus, a term $m_{3 / 2} \frac{\lambda^{2}}{4 m_{P}^{2}} \frac{1}{M_{G}} H_{u} H_{d}\left(X X^{c}\right)^{2}$ is obtained which violates PQ symmetry and hence adds a tiny correction to $\mu$. Here, $M_{G}$ is the GUT-scale Higgsino mass. Hence, PQ symmetry emerges as an approximate symmetry, thereby giving rise to a light QCD axion which does not suffer from the gravity-spoliation problem.

\section{Radiative $P Q$ breaking from SUSY breaking}

The above models are particularly compelling in that they include supersymmetry which solves the gauge hierarchy problem, but they also include the axion solution to the strong $C P$ problem of QCD. In addition, they allow for the required little hierarchy of $\mu \ll m_{\text {soft }}$. A drawback to the KN model is that it inputs the PQ scale "by hand" via the superpotential Eq. (20). It is desirable if the PQ scale can be generated via some mechanism and furthermore, the emergence of three intermediate mass scales in nature- the hidden-sector SUSY-breaking scale, the PQ scale and the Majorana neutrino scale-begs for some common origin. A model which accomplishes this was first proposed by Murayama, Suzuki and Yanagida [30].

In radiative PQ-breaking models, the MSSM superpotential is

$$
\begin{aligned}
W_{\mathrm{MSSM}}= & \sum_{i, j=1}^{3}\left[\left(\mathbf{f}_{u}\right)_{i j} Q_{i} H_{u} U_{j}^{c}+\left(\mathbf{f}_{d}\right)_{i j} Q_{i} H_{d} D_{j}^{c}\right. \\
& \left.+\left(\mathbf{f}_{e}\right)_{i j} L_{i} H_{d} E_{j}^{c}+\left(\mathbf{f}_{\nu}\right)_{i j} L_{i} H_{u} N_{j}^{c}\right]
\end{aligned}
$$

where we explicitly include the right-handed neutrino superfields $N_{i}$ and the generation indices $i, j$ run from 1-3. To this, we add a PQ superpotential containing new PQ-charged fields $X$ and $Y$ of the form

$$
W_{\mathrm{PQ}} \ni \frac{1}{2} h_{i j} X N_{i}^{c} N_{j}^{c}+\frac{f}{m_{P}} X^{3} Y+W_{\mu}
$$

and where

$$
W_{\mu}^{\mathrm{MSY}}=\frac{g_{\mathrm{MSY}}}{m_{P}} X Y H_{u} H_{d},
$$

where the PQ charges $Q_{\mathrm{PQ}}$ (matter $)=1 / 2, Q_{\mathrm{PQ}}($ Higgs $)=-1$, $Q_{\mathrm{PQ}}(X)=-1$ and $Q_{\mathrm{PQ}}(Y)=3$. Along with the MSY superpotential terms, we include the corresponding soft SUSY-breaking terms

$$
\begin{aligned}
V_{\mathrm{MSY}} \ni & m_{X}^{2}\left|\phi_{X}\right|^{2}+m_{Y}^{2}\left|\phi_{Y}\right|^{2}+m_{N_{i}}^{2}\left|\phi_{N_{i}}\right|^{2} \\
& +\left(\frac{1}{2} h_{i} A_{i} \phi_{N_{i}}^{2} \phi_{X}+\frac{f}{m_{P}} A_{f} \phi_{X}^{3} \phi_{Y}\right. \\
& \left.+\frac{g_{\mathrm{MSY}}}{m_{P}} A_{g} H_{u} H_{d} \phi_{X} \phi_{Y}+\text { H.c. }\right) .
\end{aligned}
$$

For simplicity, we assume a diagonal coupling $h_{i j}=h_{i} \delta_{i j}$. The model may be defined as applicable at the reduced Planck scale $m_{P} \simeq 2.4 \times 10^{18} \mathrm{GeV}$ and the corresponding renormalization group equations can be found in Ref. [30] at one-loop and Ref. [33] at two-loop order. Under RG evolution, the large Yukawa coupling(s) $h_{i}$ pushes the soft mass $m_{X}^{2}$ to negative values at some intermediate mass scale resulting in the radiatively induced breakdown of 
PQ symmetry as a consequence of SUSY breaking. The scalar potential consists of the terms $V=V_{F}+V_{D}+V_{\text {soft }}$. The Higgs field directions can be ignored since these develop VEVs at much lower energy scales. Then the relevant part of the scalar potential is just

$$
V_{F} \ni \frac{|f|^{2}}{m_{P}^{2}}\left|\phi_{X}^{3}\right|^{2}+\frac{9|f|^{2}}{m_{P}^{2}}\left|\phi_{X}^{2} \phi_{Y}\right|^{2} .
$$

Augmenting this with $V_{\text {soft }}$, we minimize $V$ at a scale $Q=$ $v_{\mathrm{PQ}}$ to find the VEVs of $\phi_{X}$ and $\phi_{Y}\left(v_{X}\right.$ and $\left.v_{Y}\right)$ :

$$
\begin{aligned}
0= & \frac{9|f|^{2}}{m_{P}^{2}}\left|v_{X}^{2}\right|^{2} v_{Y}+f^{*} \frac{A_{f}^{*}}{m_{P}} v_{X}^{* 3}+m_{Y}^{2} v_{Y}, \\
0= & \frac{3|f|^{2}}{m_{P}^{2}}\left|v_{X}^{2}\right|^{2} v_{X}+\frac{18|f|^{2}}{m_{P}^{2}}\left|v_{X}\right|^{2}\left|v_{Y}\right|^{2} v_{X} \\
& +3 f^{*} \frac{A_{f}^{*}}{m_{P}} v_{X}^{* 2} v_{Y}^{*}+m_{X}^{2} v_{X} .
\end{aligned}
$$

The first of these may be solved for $v_{Y}$. Substituting the first equation into the second, we find a polynomial for $v_{X}$ which may be solved for numerically. The potential has two minima in the $v_{X}$ and $v_{Y}$ plane symmetrically located with respect to the origin. For practical purposes, we use the notation $v_{X}=$ $\left|v_{X}\right|$ and $v_{Y}=\left|v_{Y}\right|$.

The fields $\phi_{X}$ and $\phi_{Y}$ obtains VEVs $v_{X}$ and $v_{Y}$ at the intermediate mass scale, taken here to be $v_{\mathrm{PQ}}=$ $\sqrt{v_{X}^{2}+9 v_{Y}^{2}}$. The corresponding axion decay constant is given by $f_{a}=\sqrt{2} v_{\mathrm{PQ}}{ }^{11}$ A DFSZ-like axion $a$ arises as the pseudo-Goldstone boson of spontaneous PQ breaking, thus solving the strong $C P$ problem. A $\mu$ parameter, which is originally forbidden by PQ symmetry, is generated with a value

$$
\mu_{\mathrm{eff}}=g_{\mathrm{MSY}} \frac{v_{X} v_{Y}}{m_{P}}
$$

and a Majorana neutrino mass, also initially forbidden by PQ symmetry, is generated at

$$
M_{N_{i}}=\left.h_{i}\right|_{Q=v_{x}} v_{X}
$$

Since the $\mu$ term depends on an arbitrary coupling $g_{\mathrm{MSY}}$, one may obtain any desired value of $\mu$ for particular $v_{X}$ and $v_{Y}$ VEVs by suitably adjusting $g_{\mathrm{MSY}}$. However, if the required values of $g_{\mathrm{MSY}}$ are very different from unity, i.e., $g_{\mathrm{MSY}} \gg 1$ or $g_{\mathrm{MSY}} \ll 1$, we might need to introduce an additional physical scale to explain the $\mu$ term. To generate a value of $\mu=150 \mathrm{GeV}$, values of $g_{\mathrm{MSY}}$ as shown in Fig. 2

\footnotetext{
${ }^{11}$ For axion interactions, the actual decay constant is $f_{A} \equiv$ $f_{a} / N_{D W}$ where $N_{D W}$ is the domain-wall number.
}

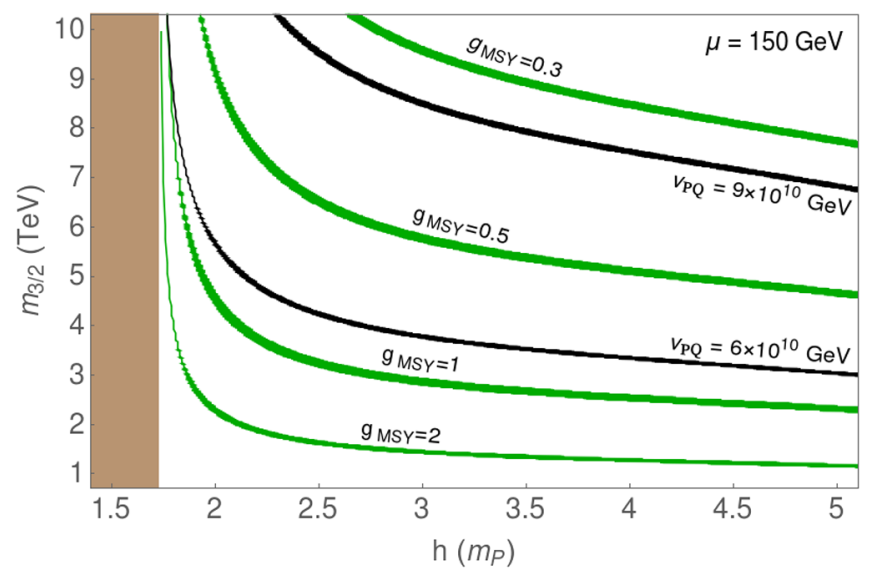

FIG. 2. Value of $g$ that is needed in the MSY to generate $\mu=$ $150 \mathrm{GeV}$ from a gravitino mass $m_{3 / 2}$ and a GUT coupling $h$. We also show some contours of $v_{\mathrm{PQ}}$.

are required depending on the values of $m_{3 / 2}$ and $h\left(M_{P}\right)$ that are assumed.

The virtues of this model include the following:

(1) It is supersymmetric, thus stabilizing the Higgs sector and allowing for a gauge hierarchy.

(2) It solves the strong $C P$ problem via a DFSZ-like axion $a$.

(3) It presents a unified treatment of the three intermediate mass scales where the PQ and Majorana neutrino scales arise as a consequence of SUSY breaking.

(4) It allows for a little hierarchy $\mu \ll m_{\text {soft }}$ for the case where $v_{\mathrm{PQ}}<m_{\text {hidden }}$.

Detailed numerical calculations in the MSY model have been carried out in Ref. [33]. There, it was found that for generic $W_{\mu}^{\mathrm{MSY}}$ couplings $g_{\mathrm{MSY}} \sim 0.1-1$, a $\mu$ parameter $\mu \sim$ $100-200 \mathrm{GeV}$ can easily be generated from TeV-scale soft breaking terms. Furthermore, since the $\mu$ term sets the mass scale for the $W, Z, h$ boson masses and is determined itself by the PQ VEVs $v_{X}$ and $v_{Y}$, the axion mass $m_{a} \simeq$ $0.48 f_{\pi} m_{\pi} / f_{a}=6.25 \times 10^{-3} \mathrm{GeV} / f_{a}$ is related to the Higgs mass $m_{h}$ and the Higgsino masses $m_{\tilde{W}_{1}, \tilde{Z}_{1,2}} \sim \mu$. The required PQ charges for the MSY model are listed in Table IX.

Other closely related models make different choices for which fields enter into $W_{\mu}$. We can also have

$$
\begin{gathered}
W_{\mu}^{\mathrm{CCK}}=\frac{g_{\mathrm{CCK}}}{m_{P}} X^{2} H_{u} H_{d} \quad \text { or } \\
W_{\mu}^{\mathrm{SPM}}=\frac{g_{\mathrm{SPM}}}{m_{P}} Y^{2} H_{u} H_{d} .
\end{gathered}
$$

The above three possibilities for $W_{\mu}$ correspond to Refs. [30] (MSY), [31] (CCK) and [32] (SPM). The corresponding PQ charges for the three radiative PQ-breaking models are listed in Table IX. 
TABLE IX. PQ charge assignments for various superfields of the CCK, MSY and SPM models of radiative PQ breaking.

\begin{tabular}{lccc}
\hline \hline Multiplet & MSY & CCK & SPM \\
\hline$H_{u}$ & -1 & -1 & -1 \\
$H_{d}$ & -1 & -1 & -1 \\
$Q$ & $+1 / 2$ & $3 / 2$ & $+1 / 2$ \\
$L$ & $+1 / 2$ & $3 / 2$ & $+5 / 6$ \\
$U^{c}$ & $+1 / 2$ & $-1 / 2$ & $+1 / 2$ \\
$D^{c}$ & $+1 / 2$ & $-1 / 2$ & $+1 / 2$ \\
$E^{c}$ & $+1 / 2$ & $-1 / 2$ & $+1 / 6$ \\
$N^{c}$ & $+1 / 2$ & $-1 / 2$ & $+1 / 6$ \\
$X$ & -1 & +1 & $-1 / 3$ \\
$Y$ & +3 & -3 & +1 \\
\hline \hline
\end{tabular}

We also show in Figs. 3 and 4 the values of $g_{\mathrm{CCK}}$ and $g_{\text {SPM }}$ that are needed to generate a value of $\mu \simeq 150 \mathrm{GeV}$. For a given value of $h\left(m_{P}\right)$ and $m_{3 / 2}$, typically $g_{\mathrm{CCK}}<$ $g_{\mathrm{MSY}}<g_{\mathrm{SPM}}$. The MSY model has the interesting feature that the PQ charge assignments are consistent with $S O(10)$ unification. We also remark that all three models can easily generate weak-scale values of $\mu$ from multi-TeV values of $m_{3 / 2}$, i.e., $\mu \ll m_{3 / 2}$ so that a little hierarchy is naturally generated.

Gravity safety of radiative PQ-breaking models: An important issue for the radiative PQ-breaking models is whether the required PQ symmetry is actually gravity safe and whether it may emerge from any of the aforementioned $\mathbb{Z}_{N}^{R}$ symmetries. We have examined whether or not the three radiative PQ-breaking models of Table IX (CCK, MSY and SPM) can be derived from any of the more fundamental $\mathbb{Z}_{N}^{R}$ symmetries in Table VIII [115]. In almost all cases, the $h X N^{c} N^{c}$ operator is disallowed; thus there is no large Yukawa coupling present to drive the PQ soft term $m_{X}^{2}$ negative so that PQ symmetry is broken. And since the PQ symmetry does not allow for a Majorana mass term

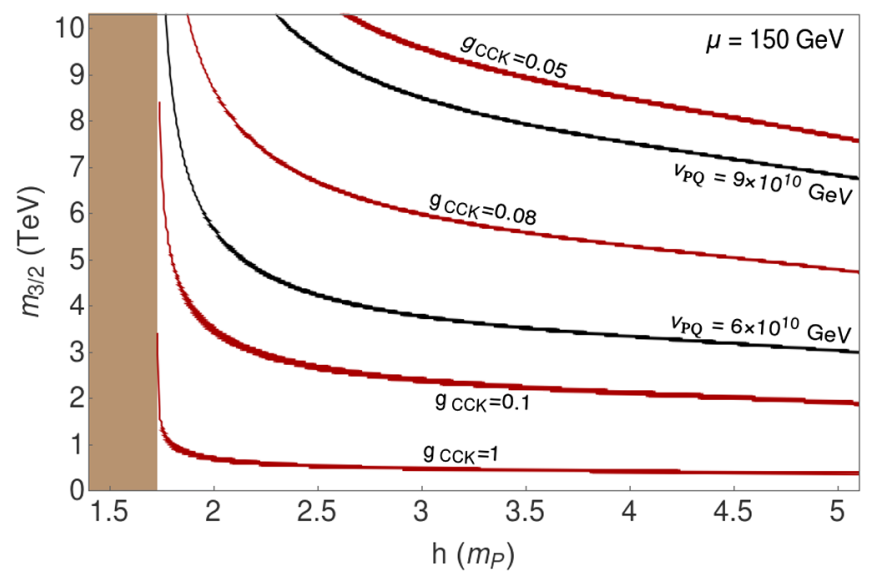

FIG. 3. Value of $g$ that is needed in the CCK to generate $\mu=$ $150 \mathrm{GeV}$ from a gravitino mass $m_{3 / 2}$ and a GUT coupling $h$. We also show some contours of $v_{\mathrm{PQ}}$.

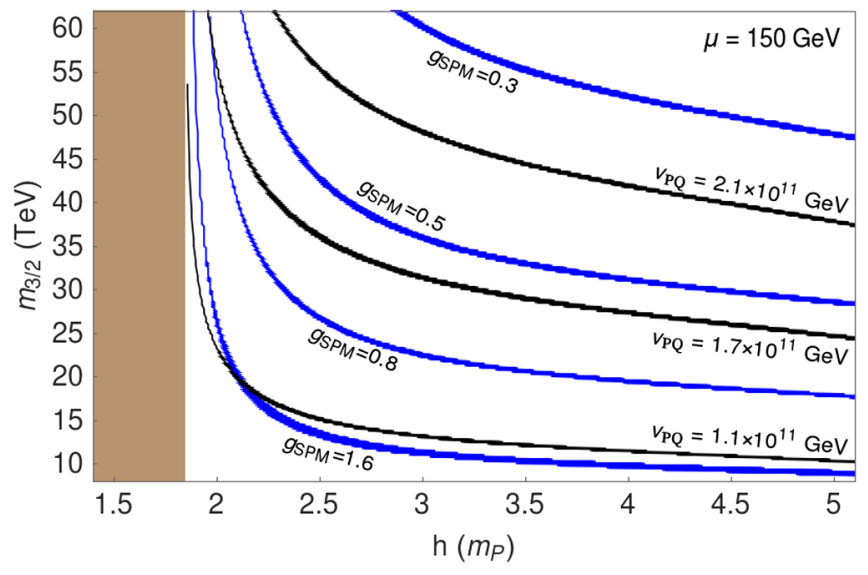

FIG. 4. Value of $g$ that is needed in the SPM model to generate $\mu=150 \mathrm{GeV}$ from a gravitino mass $m_{3 / 2}$ and a GUT coupling $h$. We also show some contours of $v_{\mathrm{PQ}}$.

$\frac{1}{2} M_{N} N^{c} N^{c}$, no seesaw scale can develop. One exception is the MSY model under $\mathbb{Z}_{4}^{R}$ symmetry with charge assignments $Q_{R}(X)=0$ and $Q_{R}(Y)=2$; then a $Y H_{u} H_{d}$ term is allowed which would generate a $\mu$ term of order the intermediate scale. Also, without considering any specific $R$ charges for the fields $X$ and $Y$, we can see that the $R$ charges for $X$ and $Y$ should be such that the term $X Y H_{u} H_{d}$ is allowed and since the $R$ charges of $H_{u}$ and $H_{d}$ are 0 , a term $M X Y$ would always be allowed: this term breaks PQ at high order and is not gravity safe. A second exception is SPM under the $\mathbb{Z}_{6}^{R}$ symmetry with charges $Q_{R}(X)=0$ and $Q_{R}(Y)=2$; then operators like $Y^{4} / m_{p}$ are allowed which break PQ but are not sufficiently suppressed so as to be gravity safe. Furthermore, we can see that in this model the $R$ charge of $Y$ is such that terms like $M^{2} Y$ which break PQ are always allowed but are not gravity safe. Thus, we conclude that while the radiative PQ-breaking models are indeed compelling and can address all three intermediate scales in a unified framework, the required PQ symmetry does not appear to be gravity safe.

\section{CCL model from gauged $U(1)_{R}$ symmetry}

In the model of Choi, Chun and Lee (CCL) [111], the $\mu$ term is generated in a manner similar to the SPM model [32], but with the difference that the fundamental symmetry is a gauged $U(1)_{R}$ symmetry out of which the PQ symmetry arises as an accidental approximate symmetry. The superpotential for the CCL model is

$$
\begin{aligned}
W_{\mathrm{CCL}}= & f_{u} Q H_{u} U^{c}+f_{d} Q H_{d} D^{c}+f_{e} L H_{d} E^{c} \\
& +f_{\nu} L H_{u} N^{c}+ \\
& +\lambda_{\mu} \frac{Y^{2} H_{u} H_{d}}{m_{p}}+\kappa X^{3} Y / m_{P}+\lambda_{N} X^{n} N^{c} N^{c} / 2 m_{P}^{n-1},
\end{aligned}
$$


TABLE X. $U(1)^{R}$ and PQ charge assignments for various superfields of the CCL model for $n=2$.

\begin{tabular}{lcccccccccc}
\hline \hline Multiplet & $H_{u}$ & $H_{d}$ & $Q_{i}$ & $L_{i}$ & $U_{i}^{c}$ & $D_{i}^{c}$ & $E_{i}^{c}$ & $N_{i}^{c}$ & $\mathrm{X}$ & $\mathrm{Y}$ \\
\hline$U(1)_{R}$ charge & 4 & 4 & $-\frac{4}{3}$ & $-\frac{4}{3}$ & $-\frac{2}{3}$ & $-\frac{2}{3}$ & $-\frac{2}{3}$ & $-\frac{2}{3}$ & $\frac{5}{3}$ & -3 \\
PQ charge & 3 & 3 & -3 & -2 & 0 & 0 & -1 & -1 & 1 & -3 \\
\hline \hline
\end{tabular}

with $U(1)_{R}$ and $P Q$ charges for the $n=2$ case given in Table X.

The singlets $X$ and $Y$ get their VEVs at the intermediate scale when the PQ symmetry is broken via a large (relative to $m_{3 / 2}$ ) negative trilinear soft term contribution to the scalar potential, thereby giving rise to $\mu \sim m_{\text {soft }}$. The $U(1)_{R}$ gauge boson has mass of order the compactification scale so the low-energy theory is that of the MSSM. Because the fundamental symmetry of CCL is a gauged $U(1)_{R}$ symmetry, the phenomenology of this model is dictated by a hierarchy of soft terms $m_{1 / 2} \gg m_{\text {scalars }}>m_{3 / 2}$ (where $m_{1 / 2}$ is the gaugino mass). Scalar soft masses are fixed in terms of $U(1)_{R} D$ terms and typically lead to large negative $m_{H_{u}}^{2}$ at the weak scale which then requires a large, unnatural $\mu$ term which would violate the $\mu \ll m_{\text {soft }}$ little hierarchy. The gravitino or the right-handed ( $\mathrm{RH})$ sneutrino turns out to be the LSP and hence ends up as a cold dark matter candidate. If the neutrino is of Majorana type then the gravitino is the LSP and if the neutrino is of Dirac type then the RH sneutrino is the LSP.

\section{MBGW model of PQ breaking from SUSY breaking}

The MBGW model $[32,39]$ begins with a superpotential

$W=f_{u} Q H_{u} U^{c}+f_{d} Q H_{d} D^{c}+f_{e} L H_{d} E^{c}+f_{\nu} L H_{u} N^{c}$

$$
+\frac{1}{2} M_{R} N^{c} N^{c}+\lambda_{\mu} \frac{X^{2} H_{u} H_{d}}{m_{p}}+\lambda_{2} \frac{(X Y)^{2}}{m_{P}}
$$

which is augmented by soft SUSY-breaking terms

$$
V_{\text {soft }} \ni m_{X}^{2}\left|\phi_{X}\right|^{2}+m_{Y}^{2}\left|\phi_{Y}\right|^{2}+\left(\lambda_{2} C \frac{\left(\phi_{X} \phi_{Y}\right)^{2}}{m_{P}}+\text { H.c. }\right)
$$

so that the scalar potential is

$$
V_{\mathrm{MBGW}}=V_{F}+V_{\mathrm{soft}}
$$

with

$$
V_{F} \ni 4 \frac{\lambda_{2}^{2}}{m_{P}}\left|\phi_{X} \phi_{Y}\right|^{2}\left(\left|\phi_{X}\right|^{2}+\left|\phi_{Y}\right|^{2}\right)
$$

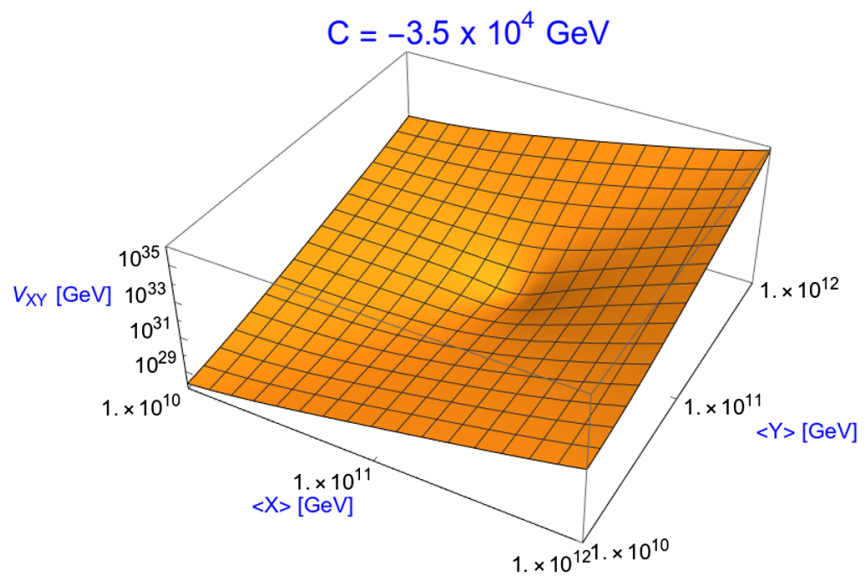

FIG. 5. Scalar potential $V_{\mathrm{MBGW}}$ vs $\phi_{X}$ and $\phi_{Y}$ for $m_{s}=$ $10^{4} \mathrm{GeV}$ and $C=-3.5 \times 10^{4} \mathrm{GeV}$.

The scalar potential admits nonzero minima in the fields $\phi_{X}$ and $\phi_{Y}$ for $C<0$. The scalar potential for the case of $m_{X}=$ $m_{Y} \equiv m_{s}=10^{4} \mathrm{GeV}$ and $C=-3.5 \times 10^{4} \mathrm{GeV}$ is shown in Fig. 5.

It was found in Ref. [39] that the model admits a remnant $\mathbb{Z}_{22}$ discrete gauge symmetry which is anomaly free up to Green-Schwarz terms and forbids lower-order operators which would lead to gravitational instability. Beside the terms in Eq. (43), the lowest-order PQ-violating term in the superpotential is $\frac{(Y)^{11}}{m_{P}^{8}}$; thus this model is gravity safe according to the KMR criterion. An approximate PQ symmetry emerges as an accidental consequence of the discrete $\mathbb{Z}_{22}$ gauge symmetry. The $\mathbb{Z}_{22}$ and PQ charges are listed in Table XI.

By taking $\left\langle\phi_{X}\right\rangle \equiv v_{x}$ and $\left\langle\phi_{Y}\right\rangle \equiv v_{Y}$, the scalar potential minimization conditions read

$0=2 \frac{\lambda_{2}}{m_{P}} C^{*} v_{x} v_{Y}^{2}+m_{X}^{2} v_{X}+4 \frac{\lambda_{2}^{2}}{m_{P}^{2}}\left(v_{X} v_{Y}^{2}\left(v_{X}^{2}+v_{Y}^{2}\right)+v_{X}^{3} v_{Y}^{2}\right)$,

$0=2 \frac{\lambda_{2}}{m_{P}} C^{*} v_{x}^{2} v_{Y}+m_{Y}^{2} v_{Y}+4 \frac{\lambda_{2}^{2}}{m_{P}^{2}}\left(v_{X}^{2} v_{Y}\left(v_{X}^{2}+v_{Y}^{2}\right)+v_{X}^{2} v_{Y}^{3}\right)$.

A simplifying assumption of $m_{X}^{2}=m_{Y}^{2} \equiv m_{s}^{2}$ and $v_{X}=$ $v_{Y} \equiv v_{s}$ leads to

TABLE XI. $\mathbb{Z}_{22}$ and PQ charge assignments for various superfields of the MBGW model.

\begin{tabular}{lrrrrrrrrrr}
\hline \hline Multiplet & $H_{u}$ & $H_{d}$ & $Q_{i}$ & $L_{i}$ & $U_{i}^{c}$ & $D_{i}^{c}$ & $E_{i}^{c}$ & $N_{i}^{c}$ & $X$ & $Y$ \\
\hline $\mathbb{Z}_{22}$ charge & 22 & 18 & 3 & 11 & 19 & 1 & 15 & 11 & 13 & 20 \\
PQ charge & -1 & -1 & +1 & +1 & 0 & 0 & 0 & 0 & +1 & -1 \\
\hline \hline
\end{tabular}




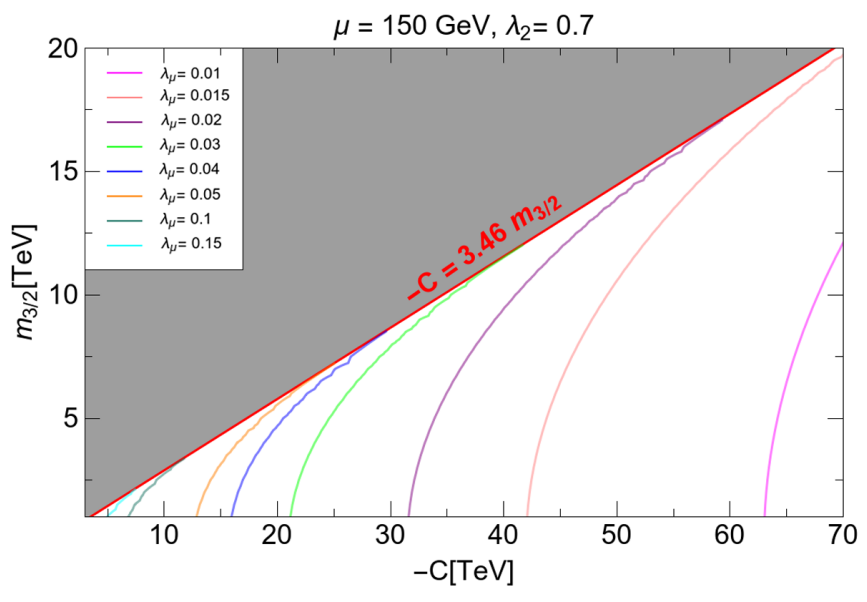

FIG. 6. Value of $\lambda_{\mu}$ required for $\mu=150 \mathrm{GeV}$ in the $m_{3 / 2}$ vs $-C$ plane of the MBGW model.

$$
v_{s}^{2}=\frac{-C \pm \sqrt{C^{2}-12 m_{s}^{2}}}{12 \lambda_{2}} m_{P}
$$

so that the $\mu$ term is

$$
\mu_{\mathrm{MBGW}} \simeq \lambda_{\mu} \frac{v_{s}^{2}}{m_{P}}
$$

with $v_{s}^{2} \simeq \frac{|C|}{12 \lambda_{2}} m_{P}$. Taking $m_{s} \simeq m_{3 / 2}=10^{4} \mathrm{GeV}$ with $\mu=$ $150 \mathrm{GeV}$ and $C=-3.5 \times 10^{4} \mathrm{GeV}$ leads to $v_{s} \simeq v_{\mathrm{PQ}} \simeq$ $10^{11} \mathrm{GeV}$ for $\lambda_{2}=0.7$ and $\lambda_{\mu} \simeq 0.036$. Thus, the MBGW model admits a little hierarchy $\mu \ll m_{3 / 2}$ while generating the PQ scale $v_{\mathrm{PQ}} \sim 10^{11} \mathrm{GeV}$ (which generates mainly axion dark matter with a smaller portion of Higgsino-like WIMPs [93,94,97]). The allowed range of the MBGW model parameter space is shown in Fig. 6 where we show contours of $\lambda_{\mu}$ values which lead to $\mu=150 \mathrm{GeV}$.

As mentioned previously, the MBGW model appears to be gravity safe under the $\mathbb{Z}_{22}$ discrete gauge symmetry. The discrete gauge symmetry $\mathbb{Z}_{M}$ might arise if a charge $M e$ field (where $M$ is a large positive integer) condenses and is integrated out of the low-energy theory while charge $e$ fields survive (see Ref. [115]). While the ensuing lowenergy theory should be gravity safe, for the case at hand one might wonder about the plausibility of the condensation of a charge 22 object and whether it might occupy the so-called swampland [116] of theories not consistent with a UV completion in string theory. In addition, the charge assignments [39] are not consistent with $S U(5)$ or $S O(10)$ grand unification which may be expected at some level in a more ultimate theory.

Alternatively, it is worth checking whether MBGW is gravity safe under any of the discrete $R$ symmetries listed in Table VIII. To check gravity safety, we note that additional superpotential terms of the form $\lambda_{3} X^{p} Y^{q}$ may be allowed for given $\mathbb{Z}_{N}^{R}$ charge assignments and powers $p$ and $q$. Such terms will typically break the PQ symmetry and render the model not gravity safe if the scalar potential $V(\phi)$ includes terms which are not suppressed by at least eight powers of $1 / m_{P}$ [38]. The largest dangerous scalar potential terms develop from interference between $\lambda_{2}(X Y)^{2} / m_{P}$ and $\lambda_{3} X^{p} Y^{q} / m_{P}^{p+q-3}$ when constructing the scalar potential $V_{F}=\sum_{\hat{\phi}}|\partial W / \partial \hat{\phi}|_{\hat{\phi} \rightarrow \phi}^{2}$ (here, $\hat{\phi}$ labels chiral superfields with $\phi$ being their leading components). We find the MBGW model to be not gravity safe under any of the $\mathbb{Z}_{N}^{R}$ discrete $R$ symmetries of Table VIII.

\section{E. Hybrid models of PQ breaking from SUSY breaking}

In this subsection, we review three models which combine approaches where PQ symmetry breaking is triggered by SUSY breaking and where a gravity-safe accidental approximate PQ symmetry might emerge from a discrete $R$ symmetry.

(1) These models are obtained by adopting a hybrid approach [115] between the radiative-breaking models and the MBGW model.

(2) In the radiative-breaking models, a Majorana neutrino scale is generated as the PQ field $X$ gets a VEV. However, in the hybrid models, the Majorana mass term $M N^{c} N^{c} / 2$ is allowed but it is not generated through PQ breaking, similar to the MBGW model.

(3) In the radiative-breaking models, intermediate PQ and Majorana neutrino scales develop as a consequence of intermediate-scale SUSY breaking and the running of the soft SUSY-breaking mass term to negative squared values. In contrast, in the MBGW model and in the hybrid models, PQ breaking is triggered by large negative soft terms instead of radiative breaking.

The three hybrid models are listed below.

\section{Hybrid CCK model}

The superpotential for the hybrid CCK model (hyCCK) is given by [115]

$$
\begin{aligned}
W_{\text {hyCCK }} \ni & f_{u} Q H_{u} U^{c}+f_{d} Q H_{d} D^{c}+f_{\ell} L H_{d} E^{c} \\
& +f_{\nu} L H_{u} N^{c}+M_{N} N^{c} N^{c} / 2 \\
& +f X^{3} Y / m_{P}+\lambda_{\mu} X^{2} H_{u} H_{d} / m_{P} .
\end{aligned}
$$

Thus when the PQ symmetry breaks, the $\mu$ parameter is obtained as

$$
\mu_{\mathrm{eff}}=\lambda_{\mu}\langle X\rangle^{2} / m_{P} .
$$

We have checked that the hyCCK model is not gravity safe under the $\mathbb{Z}_{N}^{R}$ symmetries for $N=4,6,8$ or 12 . However, it does turn out to be gravity safe under $\mathbb{Z}_{24}^{R}$ symmetry with the $\mathbb{Z}_{24}^{R}$ charge and PQ charge assignments as shown in Table XII. 
TABLE XII. $\mathbb{Z}_{24}^{R}$ and PQ charge assignments for various superfields of the hyCCK model.

\begin{tabular}{lcccccccccc}
\hline \hline Multiplet & $H_{u}$ & $H_{d}$ & $Q_{i}$ & $L_{i}$ & $U_{i}^{c}$ & $D_{i}^{c}$ & $E_{i}^{c}$ & $N_{i}^{c}$ & $\mathrm{X}$ & $\mathrm{Y}$ \\
\hline $\mathbb{Z}_{24}^{R}$ charge & 16 & 12 & 5 & 9 & 5 & 9 & 5 & 1 & -1 & 5 \\
PQ charge & -1 & -1 & 1 & 1 & 0 & 0 & 0 & 0 & 1 & -3 \\
\hline \hline
\end{tabular}

The scalar potential for hyCCK is found to be

$$
\begin{aligned}
V= & {\left[f A_{f} \frac{\phi_{X}^{3} \phi_{Y}}{m_{P}}+\text { H.c. }\right]+m_{X}^{2}\left|\phi_{X}\right|^{2}+m_{Y}^{2}\left|\phi_{Y}\right|^{2} } \\
& +\frac{f^{2}}{m_{P}^{2}}\left[9\left|\phi_{X}\right|^{4}\left|\phi_{Y}\right|^{2}+\left|\phi_{X}\right|^{6}\right]
\end{aligned}
$$

and is shown in Fig. 7 vs the scalar field values $\phi_{X}$ and $\phi_{Y}$. For large negative values of the soft term $A_{f}$, a $\mathbb{Z}_{24}^{R}$ and PQ-breaking minimum develops.

The lowest-order PQ-violating terms in the superpotential are $X^{8} Y^{2} / m_{P}^{7}, X^{4} Y^{6} / m_{P}^{7}$ and $Y^{10} / m_{P}^{7}$ which implies that the lowest-order PQ-breaking term in the scalar potential is suppressed by $1 / m_{P}^{8}$. Therefore, this model satisfies the KMR condition for being gravity safe.

The allowed range of the hyCCK model parameter space is shown in Fig. 8 where we show contours of $\lambda_{\mu}$ values which lead to $\mu=200 \mathrm{GeV}$ in the $m_{3 / 2}$ vs $-A_{f}$ plane for $f=1$. We also show several representative contours of $v_{\mathrm{PQ}}$ values. Values of $\lambda_{\mu} \sim 0.015-0.2$ are generally sufficient for a natural $\mu$ term and are easily consistent with a soft mass $m_{\text {soft }} \sim m_{3 / 2} \sim 2-30 \mathrm{TeV}$ as indicated by LHC searches. We also note that for $m_{3 / 2} \sim 5-20 \mathrm{TeV}, v_{\mathrm{PQ}} \sim 10^{11} \mathrm{GeV}$ which corresponds to the sweet spot for axion cold dark matter.

\section{Hybrid SPM Model}

The superpotential for the hybrid SPM model (hySPM) is given by $[111,115]$

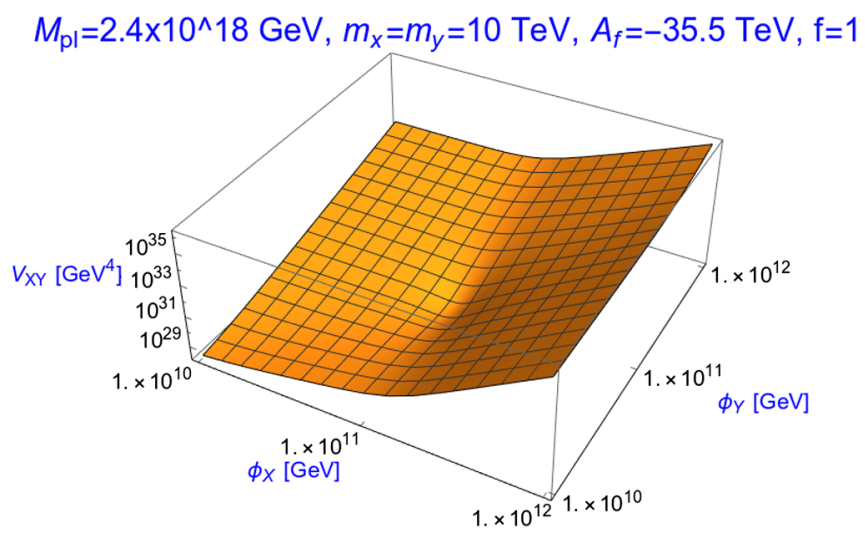

FIG. 7. Scalar potential $V_{\text {hyCCK }}$ vs $\phi_{X}$ and $\phi_{Y}$ for $m_{X}=m_{Y} \equiv$ $m_{3 / 2}=10 \mathrm{TeV}, f=1$ and $A_{f}=-35.5 \mathrm{TeV}$.

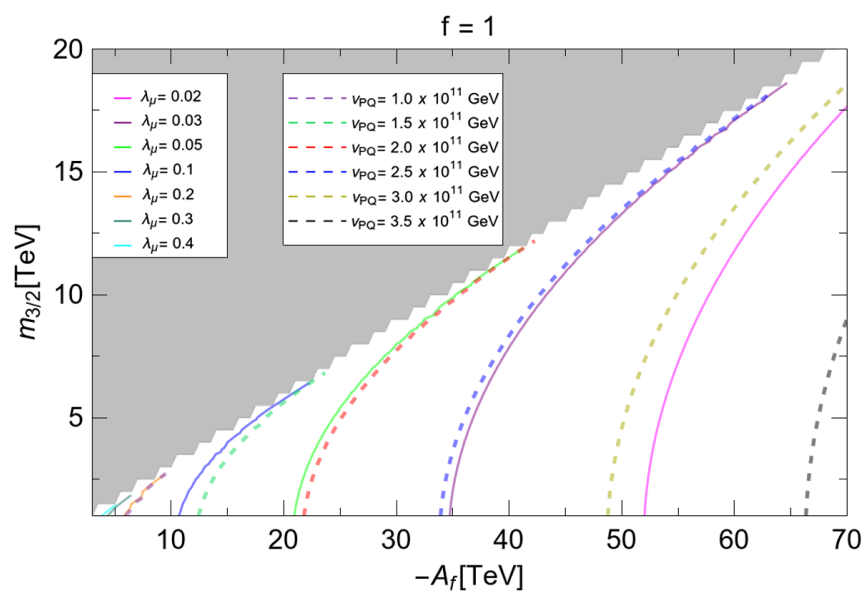

FIG. 8. Representative values of $\lambda_{\mu}$ required for $\mu=200 \mathrm{GeV}$ in the $m_{3 / 2}$ vs $-A_{f}$ plane of the hyCCK model for $f=1$. We also show several contours of $v_{\mathrm{PQ}}$.

$$
\begin{aligned}
W_{\text {hySPM }} \ni & f_{u} Q H_{u} U^{c}+f_{d} Q H_{d} D^{c}+f_{\ell} L H_{d} E^{c} \\
& +f_{\nu} L H_{u} N^{c}+M_{N} N^{c} N^{c} / 2 \\
& +f X^{3} Y / m_{P}+\lambda_{\mu} Y^{2} H_{u} H_{d} / m_{P} .
\end{aligned}
$$

In this case, when PQ symmetry breaks, the $\mu$ parameter is generated to be

$$
\mu_{\mathrm{eff}}=\lambda_{\mu}\langle Y\rangle^{2} / m_{P}
$$

This model also turns out to be not gravity safe under $\mathbb{Z}_{N}^{R}$ symmetries for $N=4,6,8$ and 12 but is gravity safe for $\mathbb{Z}_{24}^{R}$ symmetry. The gravity-safe $\mathbb{Z}_{24}^{R}$ charge and PQ charge assignments are shown in Table XIII.

The scalar potential is obtained similar to that in the hyCCK model with the only difference being that now the lowest-order PQ-violating terms in the superpotential are $Y^{8} X^{2} / m_{P}^{7}, Y^{4} X^{6} / m_{P}^{7}$ and $X^{10} / m_{P}^{7}$ which means that the lowest-order PQ-breaking terms in the scalar potential are suppressed by $1 / m_{P}^{8}$ so that the hySPM model also satisfies the KMR condition for being gravity safe.

The allowed range of the hySPM model parameter space is shown in Fig. 9 where we show contours of $\lambda_{\mu}$ values which lead to $\mu=150 \mathrm{GeV}$ in the $m_{3 / 2}$ vs $-A_{f}$ plane for $f=1$. We also show several representative contours of $v_{\mathrm{PQ}}$ values.

\section{Hybrid MSY model}

The superpotential in the hybrid MSY model (hyMSY) is given as [115]

TABLE XIII. $\mathbb{Z}_{24}^{R}$ and PQ charge assignments for various superfields of the hySPM model.

\begin{tabular}{lcccccccccc}
\hline \hline Multiplet & $H_{u}$ & $H_{d}$ & $Q_{i}$ & $L_{i}$ & $U_{i}^{c}$ & $D_{i}^{c}$ & $E_{i}^{c}$ & $N_{i}^{c}$ & $\mathrm{X}$ & $\mathrm{Y}$ \\
\hline $\mathbb{Z}_{24}^{R}$ charge & 16 & 12 & 5 & 9 & 5 & 9 & 5 & 1 & 5 & -13 \\
PQ charge & -1 & -1 & 1 & 1 & 0 & 0 & 0 & 0 & $-1 / 3$ & 1 \\
\hline \hline
\end{tabular}




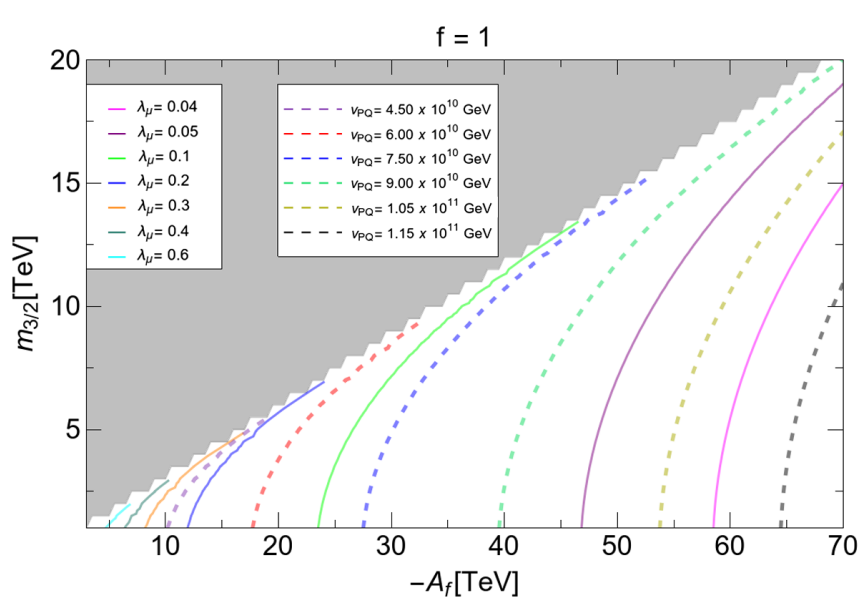

FIG. 9. Representative values of $\lambda_{\mu}$ required for $\mu=150 \mathrm{GeV}$ in the $m_{3 / 2}$ vs $-A_{f}$ plane of the hySPM model for $f=1$. We also show several contours of $v_{\mathrm{PQ}}$.

$$
\begin{aligned}
W_{\text {hyMSY }} \ni & f_{u} Q H_{u} U^{c}+f_{d} Q H_{d} D^{c}+f_{\ell} L H_{d} E^{c} \\
& +f_{\nu} L H_{u} N^{c}+M_{N} N^{c} N^{c} / 2 \\
& +f X^{3} Y / m_{P}+\lambda_{\mu} X Y H_{u} H_{d} / m_{P} .
\end{aligned}
$$

However, we have checked that the hyMSY model does not satisfy the KMR condition for being gravity safe under any of the $R$ symmetries listed in Table VIII.

\section{ARE THE VARIOUS $\mu$ SOLUTIONS EXPERIMENTALLY DISTINGUISHABLE?}

An important question arises: are the various solutions to the SUSY $\mu$ problem experimentally testable and experimentally distinguishable from one another?

Obviously, one important consequence is the existence of weak-scale SUSY (WSS) so that if WSS is disproved, then the whole discussion on the origin of the $\mu$ term is moot. The main raison d'etre for SUSY is to stabilize the weak scale under the presence of quantum corrections. In addition, WSS provides a natural mechanism for electroweak symmetry breaking. This means that no severe finetuning of parameters is involved in determining the magnitude of the weak scale, which we take to be no fine-tuning in Eq. (1). Upper limits have been derived on sparticle masses within the context of unified SUSY models with no fine-tuning $[9,15,117]$ (i.e., $\Delta_{\mathrm{EW}} \lesssim 30$ ). ${ }^{12}$ These imply typically $m_{\tilde{g}} \lesssim 6 \mathrm{TeV}$ and $m_{\tilde{t}_{1}} \lesssim 3 \mathrm{TeV}$ and $|\mu| \lesssim 360 \mathrm{GeV}$. To explore such high sparticle masses, about $15 \mathrm{ab}^{-1}$ of $p p$ collisions at $\sqrt{s} \gtrsim 27 \mathrm{TeV}$ is required

\footnotetext{
${ }^{12}$ Upper limits on sparticle masses from naturalness have also been derived in Ref. [118] using the $\Delta_{\mathrm{BG}}$ measure and Ref. [119] using the high-scale (HS) measure $\Delta_{\mathrm{HS}}$. These latter limits are much lower than those obtained using $\Delta_{\mathrm{EW}}$. However, the procedure for obtaining the BG and HS limits has been challenged in Refs. [11-14].
}

for a hadron collider [117] or $\sqrt{s} \gtrsim 720 \mathrm{GeV}$ is needed for an $e^{+} e^{-}$collider [120]. If no sparticles are seen at such colliders, then SUSY as we understand it would no longer be a viable hypothesis for stabilization of the weak scale.

Some of the $\mu$ solutions are expected to only give rise to the MSSM as the weak-scale effective theory. In this case, it may be difficult to distinguish for instance a GM solution from a $\mathrm{CM}$ solution. In the case of the G2MSSM solution, distinctive mass relations amongst sparticles are expected to occur which could support or deter such explanations [121].

In addition to weak-scale SUSY, several models- KN, CKN, EWK, HFD, radiative PQ models (MSY, CCK, SPM), MBGW and hybrid models - predict a SUSY DFSZ axion. Recent searches for axions at axion haloscope experiments [122] have reached the non-SUSY DFSZ coupling strengths for a narrow range of $m_{a}$ possibilities. However, the SUSY DFSZ axion-by virtue of including

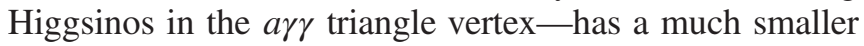
coupling [97]. It is not clear whether present technology

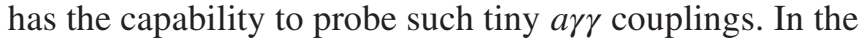
event that a thorough search can be made for SUSY DFSZ axions over their allowed range of masses and couplings strengths, then the (non)observation of axions could rule out or verify this class of $\mu$-problem solutions. A related test could be the determination of a diminished abundance of Higgsino-like WIMPs such that the presence (or absence) of additional dark matter particles such as axions is required.

Several of the $\mu$ solutions require as well additional distinctive particles. The NMSSM solution requires the presence of additional scalar and pseudoscalar Higgs bosons and a fifth neutralino arising from the NMSSM singlino. For many NMSSM parameter choices, some deviations in the $h$ boson coupling strengths are expected $[64,68]$.

The $U(1)^{\prime} \mu$ solutions also include distinctive new particle predictions. The CDEEL model [81] requires the presence of an additional weak-scale $Z^{\prime}$ boson which could decay to Higgsinos as well as SM particles [84,85]. For the HPT model [82], the $Z^{\prime}$ is expected to be far beyond any collider reach projections. Instead, for HPT, one expects bilinear RPV leading to distinctive collider signatures and altered expectations for dark matter. Also, in these models one may expect the presence of stable weak-scale exotic hadrons or other exotica which arise from the requirement for anomaly cancellation.

\section{CONCLUSIONS}

In this paper, we have reexamined the SUSY $\mu$ problem with perspective gained from experimental results from the LHC through Run 2 with $150 \mathrm{fb}^{-1}$ of data. The two parts to the SUSY $\mu$ solutions are to first 1) forbid the $\mu$ term, perhaps via some symmetry and then 2 ) regenerate it, perhaps via symmetry breaking. The new perspective from the LHC and the naturalness issue is that $\mu$ should be generated of order $m_{\text {weak }} \sim m_{W, Z, h} \sim 100-300 \mathrm{GeV}$ while 
TABLE XIV. Summary of the 20 solutions to the SUSY $\mu$ problem and 1) how they admit a LH, 2) how they solve the strong $C P$ problem $(\sqrt{ })$ or not $(\times), 3)$ whether they are expected to be gravity safe, 4$)$ whether they include the standard neutrino seesaw (SNSS) or another mechanism and 5) some experimental consequences.

\begin{tabular}{lccccc}
\hline \hline Model & & Strong & Gravity & & \\
GM & Admit LH? & $C P ?$ & safe? & Seesaw? & Exp. cons. \\
CM & small $\lambda_{\mu}$ & $\times$ & -- & SNSS & MSSM \\
$R$ sym & small $\lambda_{\mu}$ & $\times$ & -- & SNSS & MSSM \\
$\mathbb{Z}_{4}^{R}$ & $\left(v_{i} / m_{P}\right)^{n_{i}}<1$ & $\times$ & $?$ & SNSS & MSSM \\
Instanton & small $\lambda_{\mu}$ & $\times$ & -- & SNSS & MSSM \\
$G_{2}$ MSSM & small $e^{-S_{c l}}$ & $\times$ & -- & SNSS & MSSM \\
NMSSM & $\left\langle S_{i} / m_{P} \ll 1\right.$ & $\times$ & -- & SNSS & $G_{2}$ MSSM \\
nMSSM & small $\lambda_{\mu}$ & $\times$ & -- & SNSS & Extra Higgs/neutralino \\
$\mu \nu S S M$ & small $\lambda_{\mu}$ & $\times$ & -- & SNSS & Extra Higgs/neutralino \\
$U(1)^{\prime}(\mathrm{CDEEL})$ & small $\lambda_{\mu}$ & $\times$ & -- & bRPV & bRPV, mixings \\
sMSSM & small $\lambda_{\mu}$ & $\times$ & -- & SNSS & $Z^{\prime}$ \\
$U(1)^{\prime}(\mathrm{HPT})$ & small $\lambda_{\mu}$ & $\times$ & -- & SNSS & Extra Higgs/neutralino \\
KN & small $\lambda_{\mu}$ & $\times$ & -- & bRPV & bRPV, stable heavy hadrons \\
CKN & $v_{\mathrm{PQ}}<m_{\text {hidden }}$ & $\checkmark$ & $?$ & SNSS & DFSZ axion \\
BK/EWK & $\Lambda<\Lambda_{h}$ & $\checkmark$ & $?$ & SNSS & DFSZ axion \\
HFD & $\lambda_{\mu} \sim 10^{-10}$ & $\checkmark$ & $?$ & SNSS & DFSZ axion \\
MSY/CCK/SPM & $v_{\mathrm{PQ}}<m_{\text {hidden }}$ & $\checkmark$ & $?$ & SNSS & MSSM \\
CCL & $v_{\mathrm{PQ}}<m_{\text {hidden }}$ & $\checkmark$ & $\times$ & RadSS & DFSZ axion \\
BGW & small $\lambda_{\mu}$ & $\checkmark$ & $?$ & several & DFSZ axion, $\tilde{G}$ or $\tilde{\nu}$ LSP \\
Hybrid CCK/ & small $\lambda_{\mu}$ & $\checkmark$ & $\mathbb{Z}_{22}$ & SNSS & DFSZ axion \\
SPM & small $\lambda_{\mu}$ & $\checkmark$ & $\mathbb{Z}_{24}^{R}$ & SNSS & DFSZ axion \\
\hline \hline & & & & \\
\hline
\end{tabular}

the soft SUSY-breaking terms likely inhabit the multi-TeV regime. Thus, a LH should now be included in SUSY $\mu$ solutions where $|\mu| \ll m_{\text {soft }}$. This is different from preLHC expectations where solutions sought to generate $|\mu| \simeq m_{\text {soft }}$.

To gain an updated perspective on the SUSY $\mu$ problem, we examined 20 solutions. These solutions are summarized in Table XIV where we list each solution and how it may admit a LH, whether it also addresses the strong $C P$ problem, whether it is gravity safe, its relation to neutrino masses (standard seesaw or other) and any distinctive experimental consequences. While all solutions have the capacity to be consistent with the LH (usually by adjusting some arbitrary constant $\lambda_{\mu}$ ), some actually generate $\mu \sim m_{\text {weak }} \ll m_{\text {soft }}$ with $\lambda_{\mu} \sim 1$ (such as the radiative PQ-breaking models MSY, CCK and SPM).

Also, early attempts to solve the SUSY $\mu$ problem could appeal to an underlying global symmetry such as PQ to suppress the $\mu$ term. It soon became clear that such global symmetries are not consistent with an ultraviolet completion which includes gravity effects since gravitational interactions do not respect global symmetries. Continuous $\left[U(1)^{\prime}\right]$ or discrete gauge symmetries are gravity safe but usually require the addition of perhaps unwanted exotica in order to preserve anomaly freedom. The more recent emergence of discrete $R$ symmetries [40,41], which can arise from the compactification of extra dimensions in string theory, seems to provide the cleanest suppression symmetry for the $\mu$ term. A delineation of anomaly-free (including a Green-Schwarz term) $\mathbb{Z}_{N}^{R}$ symmetries which are consistent with $S O(10)$ or $S U(5)$ unification (thus preserving gauge coupling unification) offers perhaps the most compelling solutions for the first half of the SUSY $\mu$ problem. For $N=4,6,8,12$ and 24 , these symmetries forbid $\mu$ along with RPV trilinear terms and dimensionfive $p$-decay operators while allowing the required Yukawa couplings and neutrino mass operators. Of these, the $\mathbb{Z}_{4}^{R}$ stands out as both simple and compelling. It should probably now replace $R$ parity as a standard pillar upon which the MSSM is constructed.

If one also seeks to simultaneously solve the strong $C P$ problem, then the $\mathbb{Z}_{24}^{R}$ symmetry works in the hybrid models to suppress unwanted superpotential terms while providing the underlying fundamental symmetry from which a global PQ can emerge as an accidental, approximate symmetry which is gravity safe. Several other solutions also have their roots in stringy behavior [CM, $U(1)^{\prime}$, instanton, G2MSSM].

If the naturalness edict is followed-which requires $|\mu|$ to be not too far from $m_{\text {weak }} \sim 100 \mathrm{GeV}$ - then one expects thermally underproduced Higgsino-like WIMPs as (part of) dark matter. If the natural WIMP abundance is enhanced by nonthermal processes to make up the entirety of dark matter, then they become excluded by a combination of direct and indirect WIMP detection experiments [123]. Thus, additional dark matter beyond WIMPs then seems to 
be required. The axion is a highly motivated candidate to make up the remaining bulk of dark matter. To gain accord with the requirements of cold dark matter, a gravitysafe solution to the strong $C P$ problem and a solution to the SUSY $\mu$ problem (while also suppressing dangerous $p$-decay operators and allowing for seesaw neutrino masses), the hybrid models based on $\mathbb{Z}_{24}^{R}$ discrete $R$ symmetry stand out as a rather complete answer.

Overall, the SUSY $\mu$ problem has generated a rich panoply of solutions over the past 35 years. To begin the process of selecting amongst them or building others, it is of the essence to first discover SUSY and then to proceed with precision measurements of the SUSY spectra along with any exotica to gain insight into which if any of the solutions best describes nature. Future collider and dark matter experiments should go a long way towards selecting amongst or ruling out these various solutions and other solutions perhaps yet to come.

\section{ACKNOWLEDGMENTS}

We thank H. Serce for help in the early stages of this project. This work was supported in part by the US Department of Energy, Office of High Energy Physics. The work of K. J. B. was supported by Institute for Basic Science under the project code, IBS-R018-D1.
[1] E. Witten, Nucl. Phys. B188, 513 (1981); R. K. Kaul, Phys. Lett. B 109, 19 (1982).

[2] H. Baer and X. Tata, Weak Scale Supersymmetry: From Superfields to Scattering Events (Cambridge University Press, Cambridge, England, 2006), p. 537; M. Drees, R. Godbole, and P. Roy, Hackensack (World Scientific, Singapore, 2004), p. 555; S. P. Martin, Adv. Ser. Dir. High Energy Phys. 21, 1 (2010); D. J. H. Chung, L. L. Everett, G. L. Kane, S. F. King, J. D. Lykken, and L. T. Wang, Phys. Rep. 407, 1 (2005).

[3] S. Dimopoulos, S. Raby, and F. Wilczek, Phys. Rev. D 24, 1681 (1981); U. Amaldi, W. de Boer, and H. Furstenau, Phys. Lett. B 260, 447 (1991); J. R. Ellis, S. Kelley, and D. V. Nanopoulos, Phys. Lett. B 260, 131 (1991); P. Langacker and M. x. Luo, Phys. Rev. D 44, 817 (1991).

[4] L. E. Ibanez and G. G. Ross, Phys. Lett. 110B, 215 (1982); K. Inoue, A. Kakuto, H. Komatsu, and S. Takeshita Prog. Theor. Phys. 68, 927 (1982); 71, 413 (1984); L. Ibañez, Phys. Lett. 118B, 73 (1982); H. P. Nilles, M. Srednicki, and D. Wyler, Phys. Lett. 120B, 346 (1983); J. Ellis, J. Hagelin, D. Nanopoulos, and M. Tamvakis, Phys. Lett. 125B, 275 (1983); L. Alvarez-Gaumé, J. Polchinski, and M. Wise, Nucl. Phys. B221, 495 (1983); B. A. Ovrut and S. Raby, Phys. Lett. B 130, 277 (1983); for a review, see L. E. Ibanez and G. G. Ross, C.R. Phys. 8, 1013 (2007).

[5] H. E. Haber and R. Hempfling, Phys. Rev. Lett. 66, 1815 (1991); J. R. Ellis, G. Ridolfi, and F. Zwirner, Phys. Lett. B 257, 83 (1991); Y. Okada, M. Yamaguchi, and T. Yanagida, Prog. Theor. Phys. 85, 1 (1991); For a review, see e.g., M. Carena and H. E. Haber, Prog. Part. Nucl. Phys. 50, 63 (2003).

[6] H. Baer, V. Barger, and A. Mustafayev, Phys. Rev. D 85, 075010 (2012); A. Arbey, M. Battaglia, A. Djouadi, F. Mahmoudi, and J. Quevillon, Phys. Lett. B 708, 162 (2012).

[7] S. Heinemeyer, W. Hollik, D. Stockinger, A. M. Weber, and G. Weiglein, J. High Energy Phys. 08 (2006) 052.

[8] R. Barbieri and A. Strumia, arXiv:hep-ph/0007265.
[9] H. Baer, V. Barger, P. Huang, D. Mickelson, A. Mustafayev, and X. Tata, Phys. Rev. D 87, 115028 (2013).

[10] H. Baer, V. Barger, P. Huang, A. Mustafayev, and X. Tata, Phys. Rev. Lett. 109, 161802 (2012).

[11] H. Baer, V. Barger, and D. Mickelson, Phys. Rev. D 88, 095013 (2013).

[12] A. Mustafayev and X. Tata, Indian J. Phys. 88, 991 (2014).

[13] H. Baer, V. Barger, D. Mickelson, and M. PadeffkeKirkland, Phys. Rev. D 89, 115019 (2014).

[14] H. Baer, V. Barger, and M. Savoy, Phys. Scr. 90, 068003 (2015).

[15] H. Baer, V. Barger, and M. Savoy, Phys. Rev. D 93, 035016 (2016).

[16] K. L. Chan, U. Chattopadhyay, and P. Nath, Phys. Rev. D 58, 096004 (1998).

[17] H. Baer, V. Barger, and P. Huang, J. High Energy Phys. 11 (2011) 031.

[18] M. R. Douglas, arXiv:hep-th/0405279; L. Susskind, in From Fields to Strings, edited by M. Shifman et al. (World Scientific, Signapore, 2005), Vol. 3, pp. 1745-1749.

[19] H. Baer, V. Barger, M. Savoy, and H. Serce, Phys. Lett. B 758, 113 (2016); H. Baer, V. Barger, H. Serce, and K. Sinha, J. High Energy Phys. 03 (2018) 002; 04 (2019) 043.

[20] V. Agrawal, S. M. Barr, J. F. Donoghue, and D. Seckel, Phys. Rev. Lett. 80, 1822 (1998).

[21] For an early review, see e.g.,N. Polonsky, arXiv:hep-ph/ 9911329.

[22] G. F. Giudice and R. Rattazzi, Phys. Rep. 322, 419 (1999).

[23] A. Arbey, M. Battaglia, A. Djouadi, F. Mahmoudi, and J. Quevillon, Phys. Lett. B 708, 162 (2012).

[24] H. Baer, V. Barger, and A. Mustafayev, J. High Energy Phys. 05 (2012) 091.

[25] G. G. Ross, K. Schmidt-Hoberg, and F. Staub, J. High Energy Phys. 03 (2017) 021.

[26] A. E. Nelson and T. S. Roy, Phys. Rev. Lett. 114, 201802 (2015).

[27] S. P. Martin, Phys. Rev. D 92, 035004 (2015).

[28] S. K. Soni and H. A. Weldon, Phys. Lett. 126B, 215 (1983); V. S. Kaplunovsky and J. Louis, Phys. Lett. B 
306, 269 (1993); A. Brignole, L. E. Ibanez, and C. Munoz, Nucl. Phys. B422, 125 (1994); B436 (1995) 747(E); A. Brignole, L. E. Ibanez, and C. Munoz, Adv. Ser. Dir. High Energy Phys. 21, 244 (2010).

[29] J. E. Kim and H. P. Nilles, Phys. Lett. 138B, 150 (1984).

[30] H. Murayama, H. Suzuki, and T. Yanagida, Phys. Lett. B 291, 418 (1992).

[31] K. Choi, E. J. Chun, and J. E. Kim, Phys. Lett. B 403, 209 (1997).

[32] S. P. Martin, Phys. Rev. D 54, 2340 (1996); S. P. Martin, Phys. Rev. D 61, 035004 (2000); S. P. Martin, Phys. Rev. D 62, 095008 (2000).

[33] K. J. Bae, H. Baer, and H. Serce, Phys. Rev. D 91, 015003 (2015).

[34] B. Carter, The general theory of the mechanical, electromagnetic and thermodynamic properties of black holes (1979) pp. 294-369.

[35] S. B. Giddings and A. Strominger, Nucl. Phys. B306, 890 (1988); G. Gilbert, Nucl. Phys. B328, 159 (1989).

[36] R. Kallosh, A. D. Linde, D. A. Linde, and L. Susskind, Phys. Rev. D 52, 912 (1995).

[37] B. A. Dobrescu, Phys. Rev. D 55, 5826 (1997).

[38] M. Kamionkowski and J. March-Russell, Phys. Lett. B 282, 137 (1992); see also S. M. Barr and D. Seckel, Phys. Rev. D 46, 539 (1992); R. Holman, S. D. H. Hsu, T. W. Kephart, E. W. Kolb, R. Watkins, and L. M. Widrow, Phys. Lett. B 282, 132 (1992).

[39] K. S. Babu, I. Gogoladze, and K. Wang, Phys. Lett. B 560, 214 (2003).

[40] H. M. Lee, S. Raby, M. Ratz, G. G. Ross, R. Schieren, K. Schmidt-Hoberg, and P. K. S. Vaudrevange, Phys. Lett. B 694, 491 (2011).

[41] H. M. Lee, S. Raby, M. Ratz, G. G. Ross, R. Schieren, K. Schmidt-Hoberg, and P. K. S. Vaudrevange, Nucl. Phys. B850, 1 (2011).

[42] G. F. Giudice and A. Masiero, Phys. Lett. B 206, 480 (1988).

[43] C. Pallis, arXiv:1812.10284.

[44] J. A. Casas and C. Munoz, Phys. Lett. B 306, 288 (1993).

[45] S. Ramos-Sanchez, Fortschr. Phys. 58, 748 (2010).

[46] R. Kappl, H. P. Nilles, S. Ramos-Sanchez, M. Ratz, K. Schmidt-Hoberg, and P. K. S. Vaudrevange, Phys. Rev. Lett. 102, 121602 (2009); F. Brummer, R. Kappl, M. Ratz, and K. Schmidt-Hoberg, J. High Energy Phys. 04 (2010) 006.

[47] N. Arkani-Hamed, S. Dimopoulos, and S. Kachru, arXiv: hep-th/0501082.

[48] K. Choi, E. J. Chun, and H. D. Kim, Phys. Rev. D 55, 7010 (1997).

[49] L. J. Hall, Y. Nomura, and A. Pierce, Phys. Lett. B 538, 359 (2002).

[50] M. Dine and J. Kehayias, Phys. Rev. D 82, 055014 (2010).

[51] K. S. Babu, I. Gogoladze, and K. Wang, Nucl. Phys. B660, 322 (2003).

[52] K. Harigaya, M. Ibe, K. Schmitz, and T. T. Yanagida, Phys. Rev. D 88, 075022 (2013).

[53] P. Sikivie, Phys. Rev. Lett. 48, 1156 (1982).

[54] S. E. Larsson, S. Sarkar, and P. L. White, Phys. Rev. D 55, 5129 (1997).
[55] M. Dine, F. Takahashi, and T. T. Yanagida, J. High Energy Phys. 07 (2010) 003.

[56] K. J. Bae, H. Baer, H. Serce, and Y. F. Zhang, J. Cosmol. Astropart. Phys. 01 (2016) 012.

[57] L. E. Ibanez and A. M. Uranga, J. High Energy Phys. 03 (2007) 052.

[58] D. Green and T. Weigand, arXiv:0906.0595.

[59] B. S. Acharya, K. Bobkov, G. L. Kane, J. Shao, and P. Kumar, Phys. Rev. D 78, 065038 (2008).

[60] E. Witten, arXiv:hep-ph/0201018.

[61] B. S. Acharya, G. Kane, E. Kuflik, and R. Lu, J. High Energy Phys. 05 (2011) 033.

[62] L. Randall and R. Sundrum, Nucl. Phys. B557, 79 (1999); G. F. Giudice, M. A. Luty, H. Murayama, and R. Rattazzi, J. High Energy Phys. 12 (1998) 027; J. A. Bagger, T. Moroi, and E. Poppitz, J. High Energy Phys. 04 (2000) 009.

[63] B. S. Acharya, K. Bobkov, and P. Kumar, J. High Energy Phys. 11 (2010) 105.

[64] H. P. Nilles, M. Srednicki, and D. Wyler, Phys. Lett. 120B (1983) 346; J. M. Frere, D. R. T. Jones, and S. Raby, Nucl. Phys. B222, 11 (1983); J. P. Derendinger and C. A. Savoy, Nucl. Phys. B237, 307 (1984); for a recent review, see M. Maniatis, Int. J. Mod. Phys. A 25, 3505 (2010); U. Ellwanger, C. Hugonie, and A. M. Teixeira, Phys. Rep. 496, 1 (2010); U. Ellwanger, J. F. Gunion, and C. Hugonie, J. High Energy Phys. 02 (2005) 066; V. Barger, G. Shaughnessy, and B. Yencho, Phys. Rev. D 83, 055006 (2011).

[65] J. Bagger and E. Poppitz, Phys. Rev. Lett. 71, 2380 (1993); J. Bagger, E. Poppitz, and L. Randall, Nucl. Phys. B455, 59 (1995).

[66] J. R. Ellis, K. Enqvist, D. V. Nanopoulos, K. A. Olive, M. Quiros, and F. Zwirner, Phys. Lett. B 176, 403 (1986); B. Rai and G. Senjanovic, Phys. Rev. D 49, 2729 (1994); S. A. Abel, S. Sarkar, and P. L. White, Nucl. Phys. B454, 663 (1995); J. McDonald, Nucl. Phys. B530, 325 (1998).

[67] S. A. Abel, Nucl. Phys. B480, 55 (1996); C. Panagiotakopoulos and K. Tamvakis, Phys. Lett. B 446, 224 (1999); T. Han, P. Langacker, and B. McElrath, arXiv:hep-ph/ 0402064; A. Mazumdar, K. Saikawa, M. Yamaguchi, and J. Yokoyama, Phys. Rev. D 93, 025002 (2016).

[68] C. Balazs, M. Carena, A. Freitas, and C. E. M. Wagner, J. High Energy Phys. 06 (2007) 066.

[69] L. J. Hall, D. Pinner, and J. T. Ruderman, J. High Energy Phys. 04 (2012) 131.

[70] C. Panagiotakopoulos and K. Tamvakis, Phys. Lett. B 469, 145 (1999).

[71] C. Panagiotakopoulos and A. Pilaftsis, Phys. Rev. D 63, 055003 (2001).

[72] A. Dedes, C. Hugonie, S. Moretti, and K. Tamvakis, Phys. Rev. D 63, 055009 (2001).

[73] A. Menon, D. E. Morrissey, and C. E. M. Wagner, Phys. Rev. D 70, 035005 (2004).

[74] V. Barger, P. Langacker, H. S. Lee, and G. Shaughnessy, Phys. Rev. D 73, 115010 (2006).

[75] V. Barger, P. Langacker, and G. Shaughnessy, Phys. Lett. B 644, 361 (2007).

[76] J. Cao, H. E. Logan, and J. M. Yang, Phys. Rev. D 79, 091701 (2009). 
[77] K. Ishikawa, T. Kitahara, and M. Takimoto, Phys. Rev. Lett. 113, 131801 (2014).

[78] D. E. Lopez-Fogliani and C. Munoz, Phys. Rev. Lett. 97, 041801 (2006); N. Escudero, D. E. Lopez-Fogliani, C. Munoz, and R. R. de Austri, J. High Energy Phys. 12 (2008) 099; J. Fidalgo, D. E. Lopez-Fogliani, C. Munoz, and R. R. de Austri, J. High Energy Phys. 08 (2009) 105; for a review see, C. Munoz, AIP Conf. Proc. 1200, 413 (2010).

[79] K. Y. Choi, D. E. Lopez-Fogliani, C. Munoz, and R. R. de Austri, J. Cosmol. Astropart. Phys. 03 (2010) 028.

[80] P. Ghosh and S. Roy, J. High Energy Phys. 04 (2009) 069; A. Bartl, M. Hirsch, A. Vicente, S. Liebler, and W. Porod, J. High Energy Phys. 05 (2009) 120; J. Fidalgo, D. E. Lopez-Fogliani, C. Munoz, and R. R. de Austri, J. High Energy Phys. 10 (2011) 020; P. Ghosh, D. E. LopezFogliani, V. A. Mitsou, C. Munoz, and R. R. de Austri, Phys. Rev. D 88, 015009 (2013); P. Ghosh, D. E. LopezFogliani, V. A. Mitsou, C. Munoz, and R. R. de Austri, J. High Energy Phys. 11 (2014) 102.

[81] M. Cvetic, D. A. Demir, J. R. Espinosa, L. L. Everett, and P. Langacker, Phys. Rev. D 56, 2861 (1997); 58, 119905 (E) (1998).

[82] R. S. Hundi, S. Pakvasa, and X. Tata, Phys. Rev. D 79, 095011 (2009).

[83] D. E. Morrissey and J. D. Wells, Phys. Rev. D 74, 015008 (2006).

[84] T. Cohen and A. Pierce, Phys. Rev. D 78, 055012 (2008).

[85] A. Arvanitaki, Phys. Rev. D 81, 075008 (2010).

[86] J. Erler, P. Langacker, and T. j. Li, Phys. Rev. D 66, 015002 (2002).

[87] T. Han, P. Langacker, and B. McElrath, Phys. Rev. D 70, 115006 (2004).

[88] M. Hirsch and J. W. F. Valle, New J. Phys. 6, 76 (2004).

[89] M. Dine, W. Fischler, and M. Srednicki, Phys. Lett. 104B, 199 (1981); A. R. Zhitnitsky, Yad. Fiz. 31, 497 (1980) [Sov. J. Nucl. Phys. 31, 260 (1980)].

[90] R. Dermisek and A. Mafi, Phys. Rev. D 65, 055002 (2002).

[91] N. Arkani-Hamed and S. Dimopoulos, Phys. Rev. D 65, 052003 (2002); N. Arkani-Hamed, L. Hall, D. Smith, and N. Weiner, Phys. Rev. D 63, 056003 (2001).

[92] K. Y. Choi, J.E. Kim, H. M. Lee, and O. Seto, Phys. Rev. D 77 (2008) 123501; H. Baer, A. Lessa, S. Rajagopalan, and W. Sreethawong, J. Cosmol. Astropart. Phys. 06 (2011) 031.

[93] K. J. Bae, H. Baer, and E. J. Chun, Phys. Rev. D 89, 031701 (2014); K. J. Bae, H. Baer, and E. J. Chun, J. Cosmol. Astropart. Phys. 12 (2013) 028.

[94] K. J. Bae, H. Baer, A. Lessa, and H. Serce, J. Cosmol. Astropart. Phys. 10 (2014) 082.

[95] E. J. Chun, Phys. Rev. D 84, 043509 (2011); K. J. Bae, E. J. Chun, and S. H. Im, J. Cosmol. Astropart. Phys. 03 (2012) 013.

[96] M. Tanabashi et al. (Particle Data Group), Phys. Rev. D 98, 030001 (2018).

[97] K. J. Bae, H. Baer, and H. Serce, J. Cosmol. Astropart. Phys. 06 (2017) 024.
[98] J. E. Kim and H. P. Nilles, Phys. Lett. B 263, 79 (1991); E. J. Chun, J. E. Kim, and H. P. Nilles, Nucl. Phys. B370, 105 (1992).

[99] J. E. Kim and H. P. Nilles, Phys. Lett. B 553, 1 (2003); J. E. Kim and H. P. Nilles, J. Cosmol. Astropart. Phys. 05 (2009) 010.

[100] M. Bastero-Gil and S. F. King, Phys. Lett. B 423, 27 (1998).

[101] O. J. Eyton-Williams and S. F. King, Phys. Lett. B 610, 87 (2005).

[102] A. D. Linde, Phys. Lett. B 249, 18 (1990); A. D. Linde, Phys. Lett. B 259, 38 (1991).

[103] O. J. Eyton-Williams and S. F. King, J. High Energy Phys. 06 (2005) 040.

[104] M. Dine and N. Seiberg, Nucl. Phys. B273, 109 (1986).

[105] L. M. Carpenter, M. Dine, and G. Festuccia, Phys. Rev. D 80, 125017 (2009).

[106] L. M. Krauss and F. Wilczek, Phys. Rev. Lett. 62, 1221 (1989); J. E. Kim and H. P. Nilles, Mod. Phys. Lett. A 09, 3575 (1994); T. Banks, Nucl. Phys. B323, 90 (1989).

[107] G. Lazarides, C. Panagiotakopoulos, and Q. Shafi, Phys. Rev. Lett. 56, 432 (1986).

[108] J. E. Kim and H. P. Nilles, Phys. Lett. B 730, 53 (2014).

[109] H. Baer, K. Y. Choi, J. E. Kim, and L. Roszkowski, Phys. Rep. 555, 1 (2015).

[110] E. J. Chun and A. Lukas, Phys. Lett. B 297, 298 (1992).

[111] K. Y. Choi, E. J. Chun, and H. M. Lee, Phys. Rev. D 82, 105028 (2010).

[112] A. H. Chamseddine and H. K. Dreiner, Nucl. Phys. B458, 65 (1996).

[113] M. C. Chen, M. Fallbacher, and M. Ratz, Mod. Phys. Lett. A 27, 1230044 (2012).

[114] J. E. Kim, Phys. Rev. Lett. 111, 031801 (2013).

[115] H. Baer, V. Barger, and D. Sengupta, Phys. Lett. B 790, 58 (2019).

[116] C. Vafa, arXiv:hep-th/0509212.

[117] H. Baer, V. Barger, J. S. Gainer, D. Sengupta, H. Serce, and X. Tata, Phys. Rev. D 98, 075010 (2018).

[118] J. R. Ellis, K. Enqvist, D. V. Nanopoulos, and F. Zwirner, Mod. Phys. Lett. A 01, 57 (1986); R. Barbieri and G. F. Giudice, Nucl. Phys. B306, 63 (1988); S. Dimopoulos and G. F. Giudice, Phys. Lett. B 357, 573 (1995); G. W. Anderson and D. J. Castano, Phys. Rev. D 52, 1693 (1995).

[119] M. Papucci, J. T. Ruderman, and A. Weiler, J. High Energy Phys. 09 (2012) 035; C. Brust, A. Katz, S. Lawrence, and R. Sundrum, J. High Energy Phys. 03 (2012) 103.

[120] H. Baer, V. Barger, D. Mickelson, A. Mustafayev, and X. Tata, J. High Energy Phys. 06 (2014) 172; S. L. Lehtinen et al. (ILD concept group), Proc. Sci. EPS-HEP2017 (2017) 306.

[121] B. S. Acharya, G. Kane, and P. Kumar, Int. J. Mod. Phys. A 27, 1230012 (2012).

[122] N. Du et al. (ADMX Collaboration), Phys. Rev. Lett. 120, 151301 (2018).

[123] H. Baer, V. Barger, D. Sengupta, and X. Tata, Eur. Phys. J. C 78, 838 (2018). 